\title{
Review \\ Opioid-Sparing Effect of Cannabinoids: A Systematic Review and Meta-Analysis
}

\author{
Suzanne Nielsen ${ }^{*}, 1,2$, Pamela Sabioni ${ }^{3}$, Jose M Trigo ${ }^{3}$, Mark A Ware ${ }^{4}$, Brigid D Betz-Stablein ${ }^{5}$, \\ Bridin Murnion $^{6,7}$, Nicholas Lintzeris ${ }^{2,6}$, Kok Eng Khor ${ }^{8}$, Michael Farrell', Andrew Smith' ${ }^{9}$ and Bernard Le Foll ${ }^{3}$ \\ 'The National Drug and Alcohol Research Centre, The University of New South Wales, Sydney, NSW, Australia; ' ${ }^{2}$ rug and Alcohol Services, South \\ Eastern Sydney Local Health District, Surry Hills, NSW, Australia; ${ }^{3}$ Translational Addiction Research Laboratory, Campbell Family Mental Health \\ Research Institute, Centre for Addiction and Mental Health, Toronto, ON, Canada; ${ }^{4}$ Departments of Anaesthesia and Family Medicine, McGill \\ University, Montreal, QC, Canada; ${ }^{5}$ School of Public Health and Community Medicine, The University of New South Wales, Sydney, NSW, Australia; \\ ${ }^{6}$ Discipline of Addiction Medicine, University of Sydney, Sydney, NSW, Australia; ${ }^{7}$ Pain Management Centre, Royal Prince Alfred Hospital, \\ Camperdown, NSW, Australia; ${ }^{8}$ Department of Pain Management, Prince of Wales Hospital, Randwick, NSW, Australia; ${ }^{9}$ Pain and Addiction \\ Medicine, Centre for Addiction and Mental Health, Toronto, ON, Canada
}

\begin{abstract}
Cannabinoids, when co-administered with opioids, may enable reduced opioid doses without loss of analgesic efficacy (ie, an opioidsparing effect). The aim of this study was to conduct a systematic review to determine the opioid-sparing potential of cannabinoids. Eligible studies included pre-clinical and clinical studies for which the outcome was either analgesia or opioid dose requirements. Clinical studies included controlled studies and case series. We searched Scopus, Cochrane Database of Systematic Reviews, Medline, and Embase. Nineteen pre-clinical and nine clinical studies met the search criteria. Seventeen of the 19 pre-clinical studies provided evidence of synergistic effects from opioid and cannabinoid co-administration. Our meta-analysis of pre-clinical studies indicated that the median effective dose $\left(E D_{50}\right)$ of morphine administered in combination with delta-9-tetrahydrocannabinol (delta-9-THC) is 3.6 times lower (95\% confidence interval $(\mathrm{Cl}) 1.95,6.76 ; n=6)$ than the $\mathrm{ED}_{50}$ of morphine alone. In addition, the $\mathrm{ED}_{50}$ for codeine administered in combination with delta-9-THC was 9.5 times lower $(95 \% \mathrm{Cl} 1.6,57.5, n=2)$ than the $\mathrm{ED}_{50}$ of codeine alone. One case series $(n=3)$ provided very-low-quality evidence of a reduction in opioid requirements with cannabinoid co-administration. Larger controlled clinical studies showed some clinical benefits of cannabinoids; however, opioid dose changes were rarely reported and mixed findings were observed for analgesia. In summary, pre-clinical studies provide robust evidence of the opioid-sparing effect of cannabinoids, whereas one of the nine clinical studies identified provided very-low-quality evidence of such an effect. Prospective high-quality-controlled clinical trials are required to determine the opioid-sparing effect of cannabinoids.

Neuropsychopharmacology (2017) 42, 1752-1765; doi:I0.1038/npp.2017.5I; published online 5 April 2017
\end{abstract}

\section{INTRODUCTION}

Chronic pain is associated with enormous personal, social, and economic burden and is the largest contributor to years lived with disability globally (Rice et al, 2015). Despite this, existing medications provide only modest relief. Opioids in particular have considerable side effects, including constipation, impaired sleep, and respiratory depression (Chou et al, 2015). The last two decades have seen an increase in the prescription of opioids, which has been associated with an increase in opioid use disorders and opioid-related mortality (Chou et al, 2015; Volkow and McLellan, 2016; Zedler et al, 2014). This has been termed as an 'opioid crisis', and has

* Correspondence: Dr S Nielsen, The National Drug and Alcohol Research Centre, The University of New South Wales, Sydney, NSW 2052, Australia, Tel: +6I 28936 I017, Fax: +6I 293850222 ,

E-mail: suzanne.nielsen@unsw.edu.au

Received II July 2016; revised 3I January 2017; accepted 7 March 2017; accepted article preview online 22 March 2017 caused regulators, health professionals, and the public to begin seeking means to reduce problems associated with high-dose opioid use. Consequently, there is a need for evidence-based strategies for reducing reliance on high-dose opioids without compromising pain management.

Using combinations of medications to harness complementary but distinct mechanisms of action can maximize the analgesic response, enabling the use of a lower dose of each medication and resulting in an improved side effect profile. One promising area for medication combinations is the use of opioid-sparing medications. Opioid-sparing medications, when co-administered with opioids, enable a reduced opioid dose without loss of analgesic efficacy. Cannabinoid medications are increasingly being studied for their analgesic- and opioid-sparing potential. The endocannabinoid system represents an ideal target because it is a key endogenous system in modulating pain-processing pathways (Woodhams et al, 2015).

The endocannabinoid system is composed of the cannabinoid $\mathrm{CB} 1$ and $\mathrm{CB} 2$ receptors, the endocannabinoid ligands 
anandamide and 2-arachidonoylglycerol, and their synthesis and degradation system (Pertwee, 2006). CB1 and CB2 receptors are differentially expressed on the central nervous system (Cencioni et al, 2010; Herkenham et al, 1991) and play important roles in pain processes. Both cannabinoid receptors and endocannabinoids are present in the primary afferent pain circuits to the brain (Manzanares et al, 1999; Woodhams et al, 2015). Cannabinoid and opioid receptors have similar signal transduction systems (Cichewicz, 2004; Howlett et al, 2002; Vigano et al, 2005) and are expressed in several brain regions involved in antinociception, including the periaqueductal gray, raphe nuclei, and central-medial thalamic nuclei (Cichewicz, 2004). In addition, mu-opioid receptors and CB1 receptors co-localize in the spinal cord at the first synaptic contact for peripheral nociceptive afferent neurons (Hohmann et al, 1999; Salio et al, 2001).

It has previously been observed that $\mathrm{CB} 2$ receptors indirectly stimulate opioid receptors located in primary afferent pathways (Ibrahim et al, 2005). Therefore, in addition to their direct analgesic effects, cannabinoids may work synergistically to enhance opioid analgesia. The behavioral, anatomical, and biochemical similarities between opioid and cannabinoid receptor systems and their endogenous ligands are well documented. For example, activation of either cannabinoid or opioid receptors produces comparable neurobehavioral and physiological effects, including antinociception (Manzanares et al, 1999). This is highlighted by both $\mathrm{CB} 1$ and $\mathrm{CB} 2$ agonists being able to induce antinociception by increasing opioid precursors' gene expression or via release of endogenous opioids (Houser et al, 2000; Ibrahim et al, 2005; Valverde et al, 2001). Further, pharmacological modulation of the opioid system can modify the effects of delta-9-tetrahydrocannabinol (delta-9THC) - a partial agonist at the CB1 and CB2 receptor-on nociception (Mason et al, 1999; Pugh et al, 1997; Smith et al, 1994) and vice versa. Finally, cannabinoid antagonists have been shown to reverse the antinociception induced by morphine (da Fonseca Pacheco et al, 2008). Collectively, this strongly supports shared mechanisms between both systems in regard to analgesia.

Animal models have identified a role for CB1 receptor activation in reducing neuropathic, visceral, and inflammatory pain (Pertwee, 2008; Walker et al, 1999). Several preclinical studies have demonstrated that systemic administration of cannabinoid receptor ligands produces analgesia in acute and chronic pain models (Walker and Huang, 2002). In addition, the role of $\mathrm{CB} 2$ receptors has been explored in pre-clinical studies, suggesting that these receptors may mediate effects in inflammatory pain states (Ibrahim et al, 2006; Quartilho et al, 2003), and reduce inflammation and neuropathic pain (Gui et al, 2015).

Further to these pre-clinical findings, clinical studies indicate that cannabinoid administration may reduce pain and improve other symptoms such as sleep disturbances associated with chronic pain (Ware et al, 2010a; Ware et al, 2010b). This effect could be mediated by delta-9-THC, which is the main psychoactive ingredient present in cannabis (Cichewicz, 2004; Jensen et al, 2015). Despite the growing body of relevant literature, to date no systematic review has focused on the opioid-sparing effects of cannabinoids. To address this gap, we conducted a systematic review of preclinical and clinical studies to examine the strength of existing evidence demonstrating the opioid-sparing effect of cannabinoids in the context of analgesia.

\section{MATERIALS AND METHODS}

\section{Search}

We conducted a systematic search of the literature in accordance with recommendations by the Preferred Reporting Items for Systematic Reviews and Meta-Analyses (PRISMA) (Moher et al, 2009). The search aimed to identify clinical and pre-clinical studies using the following electronic databases: Scopus, Cochrane Database of Systematic Reviews, Medline, and Embase. Search terms are listed and a sample search strategy is reported in Supplementary Appendix 1. No date limits were included. Searches were run on 29 October 2015. In addition, reference lists from identified studies and review articles were searched to find additional studies not identified by the main search.

Eligible studies included:

- Human or animal studies.

- Outcomes of either pain/analgesia or opioid requirements/ opioid-sparing effects from concurrently administered opioids and cannabinoids.

- Controlled clinical studies and case series.

Titles were screened by two authors (SN and PS). Where inconsistencies were identified, the authors were able to reach consensus on each occasion.

\section{Data Extraction and Outcomes}

Data extraction forms were developed and circulated to the author group before piloting and refining. All data were extracted by one of the authors (SN, PS, or JMT) and checked by a second author (SN, PS, or JMT). These same authors reviewed and resolved any inconsistencies, with input from the authorship group as required. When required data were missing, attempts were made to contact authors of published reports to collect additional information.

\section{Outcome Measures}

For pre-clinical studies, the primary outcome was the dose of opioid required to give an equivalent antinociceptive effect in the presence and absence of cannabinoids. For clinical studies, the primary outcome was evidence of the opioidsparing effect of cannabinoids. Data were extracted on opioid dose and/or analgesic outcome where cannabinoids were co-administered. Secondary outcome measures examined included analgesia, sleep, and quality of life.

\section{Analysis}

Pre-clinical studies. Data were extracted and a narrative review was conducted. Ten studies were identified as sufficiently similar in design and outcome measures to be eligible for meta-analysis. Of these, six reported sufficient data to enable meta-analysis; that is, the dose of opioid required to produce comparable analgesia in the presence and absence of cannabinoids, the variance of the observed dose, and the sample size. Authors of the other studies were 


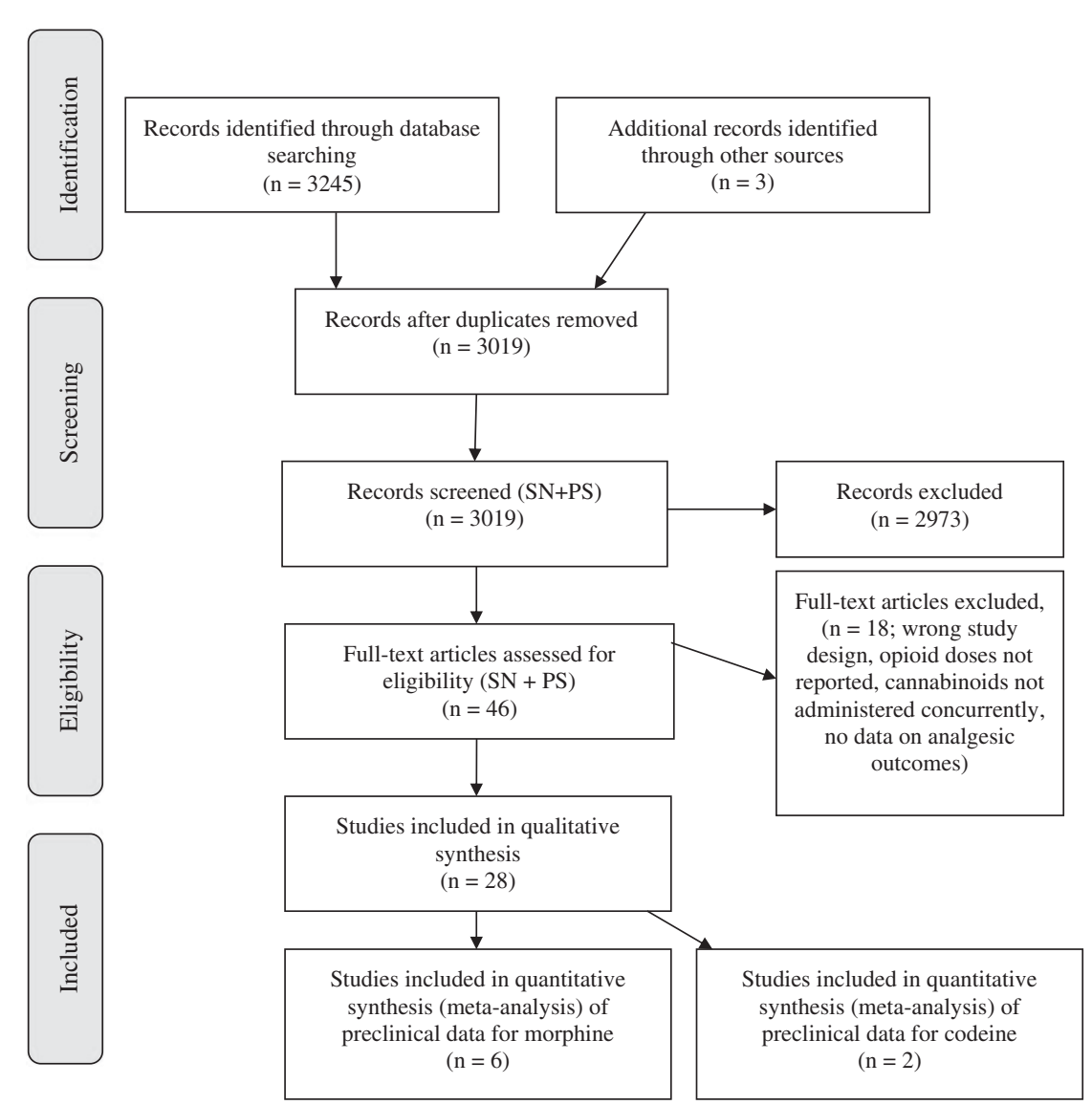

Figure I PRISMA diagram showing study identification. PRISMA, Preferred Reporting Items for Systematic Reviews and Meta-Analyses.

contacted in an attempt to include additional studies in the meta-analysis; however, no additional data were identified to enable the inclusion of any additional studies.

To prepare the data for the meta-analysis, the effective dose $\left(\mathrm{ED}_{50}\right)$ and either confidence limits or SE were extracted from the relevant literature. $\mathrm{ED}_{50}$ is calculated on the $\log _{10}$ scale. Therefore, to meet the assumption of normality, the $\log _{10} \widehat{\mathrm{ED}}_{50}$ must be used in the metaanalysis. The $\log _{10}$ of the confidence limits must also be determined to calculate the SD of the $\log _{10} \widehat{\mathrm{ED}}_{50}$ :

$\mathrm{SD}\left(\log _{10} \widehat{\mathrm{ED}}_{50}\right)=\frac{\log _{10} \mathrm{UL}-\log _{10} \widehat{\mathrm{ED}}_{50}}{1.96}$,

where UL is the upper confidence limit.

When only SE was reported, the confidence limits were calculated using the method of Litchfield and Wilcoxon (1949) and the above procedure was repeated to calculate the SD. This method also allowed for the inclusion of studies that did not report exact sample sizes for all treatment groups, as sample size was not required for the calculation of SD.

Data for the meta-analysis were analyzed using Review Manager 5.1 (Cochrane Collaboration, Oxford, UK). When calculating the continuous outcome of an equally effective opioid dose (eg, the $\log _{10} \mathrm{ED}_{50}$ for morphine when administered alone $v s$ when administered with a cannabinoid), the inverse variance statistical method and random effects model were used to compensate for study heterogeneity.
No statistical difference was found in outcomes between the studies that used different species or nociceptive assays. Therefore, the mean difference of $\log _{10} \mathrm{ED}_{50}$ of and the corresponding 95\% confidence intervals (CI) were calculated. Due to the nature of log calculations, the mean difference-when back-transformed to the original unitsrepresents the response ratio. For easier interpretation, we present the reciprocal of the response rate.

Assessment of bias in pre-clinical studies. A funnel plot was produced to examine publication bias and small study effects in the pre-clinical studies included in the metaanalysis.

Clinical studies. The nine clinical studies identified were heterogeneous in design and outcomes, and therefore not suitable for meta-analysis. Thus, a narrative synthesis was conducted instead, with all studies scored for quality using the Grading of Recommendations Assessment, Development and Evaluation (GRADE) criteria (Guyatt et al, 2008).

\section{RESULTS}

The initial searches identified 3019 records after duplicates were removed, with 19 pre-clinical and nine clinical studies identified for inclusion in the final review (see Figure 1 for the PRISMA diagram). 
Table I Summary of Evidence of Opioid-Sparing Effects from Pre-Clinical Studies

Equipotent opioid dose represented as $\mathrm{ED}_{50}(95 \% \mathrm{CL})$ or \pm SEM, unless measured otherwise specified

\begin{tabular}{|c|c|c|c|c|c|c|c|}
\hline $\begin{array}{l}\text { Study } \\
\text { reference }\end{array}$ & Pain model (species) & Opioid administered & $\begin{array}{l}\text { Cannabinoid } \\
\text { administered }\end{array}$ & Cannabinoid condition & Vehicle condition & $\begin{array}{l}\text { Potency ratio or evidence of } \\
\text { synergism }\end{array}$ & Other notes \\
\hline \multirow[t]{10}{*}{$\begin{array}{l}\text { Cichewicz et al, } \\
1999\end{array}$} & $\begin{array}{l}\text { Tail-flick test } \\
\text { (male ICR mice) }\end{array}$ & Morphine p.o. & Delta-9-THC (20 mg/kg p.o.) & 13.1 mg/kg $(8.8,19.5)$ & $28.8 \mathrm{mg} / \mathrm{kg}(20.2,41)$ & Potency ratio: 2.2 & \\
\hline & & Codeine p.o. & Delta-9-THC (20 mg/kg p.o.) & $5.9 \mathrm{mg} / \mathrm{kg}(1.4,24.9)$ & 139.9 mg/kg (75.2, 260.5) & Potency ratio: 25.8 & \\
\hline & & Oxymorphone p.o. & Delta-9-THC (20 mg/kg p.o.) & 0.5 mg/kg $(0.3,0.8)$ & $2.6 \mathrm{mg} / \mathrm{kg}(1.7,3.9)$ & Potency ratio: 5.0 & \\
\hline & & Hydromorphone p.o. & Delta-9-THC (20 mg/kg p.o.) & $0.4 \mathrm{mg} / \mathrm{kg}(0.2,0.8)$ & $5.6 \mathrm{mg} / \mathrm{kg}(3.2,9.7)$ & Potency ratio: 12.6 & \\
\hline & & Methadone p.o. & Delta-9-THC (20 mg/kg p.o.) & $2.7 \mathrm{mg} / \mathrm{kg}(1.4,5.2)$ & $12.0 \mathrm{mg} / \mathrm{kg}(8.1,17.9)$ & Potency ratio: 4.1 & \\
\hline & & LAAM р.o. & Delta-9-THC (20 mg/kg p.o.) & $2.6 \mathrm{mg} / \mathrm{kg}(1.7,3.9)$ & $8.0 \mathrm{mg} / \mathrm{kg}(6.4,10.1)$ & Potency ratio: 2.5 & \\
\hline & & Heroin p.o. & Delta-9-THC (20 mg/kg p.o.) & $5.4 \mathrm{mg} / \mathrm{kg}(1.7,16.9)$ & $26.1 \mathrm{mg} / \mathrm{kg}(12.7,53.4)$ & Potency ratio: 4.1 & \\
\hline & & Meperidine p.o. & Delta-9-THC (20 mg/kg p.o.) & $11.1 \mathrm{mg} / \mathrm{kg}(4.2,29.4)$ & $86.2 \mathrm{mg} / \mathrm{kg}(52.8,140.6)$ & Potency ratio: 8.9 & \\
\hline & & Fentanyl p.o. & Delta-9-THC (20 mg/kg p.o.) & $0.5 \mathrm{mg} / \mathrm{kg}(0.3,0.8)$ & $\begin{array}{l}6.1 \mathrm{mg} / \mathrm{kg} \text { (estimated from an } \\
\text { extrapolated curve) }\end{array}$ & $\begin{array}{l}\text { Not determined (50\% MPE not } \\
\text { seen) }\end{array}$ & \\
\hline & & Pentazocine p.o. & Delta-9-THC (20 mg/kg p.o.) & $\begin{array}{l}838.6 \mathrm{mg} / \mathrm{kg} \text { (estimated } \\
\text { from an extrapolated curve) }\end{array}$ & $\begin{array}{l}625.9 \mathrm{mg} / \mathrm{kg} \text { (estimated from } \\
\text { an extrapolated curve) }\end{array}$ & $\begin{array}{l}\text { Not determined (50\% MPE not } \\
\text { seen) }\end{array}$ & \\
\hline \multirow[t]{3}{*}{$\begin{array}{l}\text { Cichewicz and } \\
\text { Welch, } 2003\end{array}$} & $\begin{array}{l}\text { Tail-flick test } \\
\text { (male ICR mice) }\end{array}$ & $\begin{array}{l}\text { Morphine p.o. } \\
\text { Codeine p.o. }\end{array}$ & $\begin{array}{l}\text { Delta-9-THC (5-35 mg/kg } \\
\text { and I-27 mg/kg p.o.) }\end{array}$ & $13.6 \mathrm{mg} / \mathrm{kg} \pm 1.94$ & $\begin{array}{l}24.5 \mathrm{mg} / \mathrm{kg} \pm 4.8 \\
78.2 \mathrm{mg} / \mathrm{kg} \pm 14.4\end{array}$ & $\begin{array}{l}\text { For each ratio tested, experimental } \\
\text { values were less than the calculated } \\
\text { additive values (synergism) }\end{array}$ & $\begin{array}{l}\text { Fixed-ratio combinations of 9-THC } \\
\text { with either morphine or codeine } \\
\text { were tested for antinociceptive } \\
\text { effects. The experimentally derived }\end{array}$ \\
\hline & & & $\begin{array}{l}\text { Delta-9-THC (5-30 and } \\
5-18 \mathrm{mg} / \mathrm{kg} \text { p.o.) }\end{array}$ & $20.1 \mathrm{mg} / \mathrm{kg} \pm 3.0$ & & $\begin{array}{l}\text { For each ratio tested, experimental } \\
\text { values were less than the calculated } \\
\text { additive values (synergism) }\end{array}$ & $\begin{array}{l}\mathrm{ED}_{50} \text { for each combination was } \\
\text { compared with the theoretical } \\
\text { additive } \mathrm{ED}_{50} \text {, using an }\end{array}$ \\
\hline & & & & & & & $\begin{array}{l}\text { isobolographic analysis. All the } \\
\text { fixed-ratio combinations tested } \\
\text { produced greater antinociception } \\
\text { (synergy) than predicted from } \\
\text { simple additivity }\end{array}$ \\
\hline \multirow[t]{4}{*}{$\begin{array}{l}\text { Cichewicz et al, } \\
2005\end{array}$} & $\begin{array}{l}\text { Pin-prick test } \\
\text { (IAF hairless guinea pigs) }\end{array}$ & Fentanyl s.c. & Delta-9-THC (50 mg/kg i.p.) & $6.8 \mu \mathrm{gg} / \mathrm{kg}(3.3,14.2)$ & $50.8 \mu g / k g(41.0,63.0)$ & $\begin{array}{l}\text { Greater than additive effect on } \\
\text { antinociception. Potency ratio: } 6.7 \\
(1.8-17.0)\end{array}$ & \\
\hline & & Buprenorphine s.c. & Delta-9-THC (50 mg/kg i.p.) & $0.02 \mathrm{mg} / \mathrm{kg}(0.01,0.05)$ & $2.97 \mathrm{mg} / \mathrm{kg}(\mathrm{I} .84,4.8 \mathrm{I})$ & $\begin{array}{l}\text { Greater than additive effect on } \\
\text { antinociception. Enhanced potency } \\
\text { in a non-parallel fashion }\end{array}$ & $\begin{array}{l}\text { Not possible to compare the } \\
\text { change in potency produced by } \\
\text { delta-9-THC due to the non- } \\
\text { parallel nature of the two dose- } \\
\text { response curves for buprenorphine }\end{array}$ \\
\hline & & Fentanyl t.d. & Delta-9-THC (400 mg/kg t.d.) & $\begin{array}{l}2 \mathrm{h:}: 254.9 \mu \mathrm{g} / \mathrm{kg} \\
(202.90,320.6) \\
4 \mathrm{h:}: 176.3 \mu \mathrm{g} / \mathrm{kg} \\
(144.3,215.5)\end{array}$ & $\begin{array}{l}2 \mathrm{h:}: 928.6 \mathrm{\mu g} / \mathrm{kg} \\
(599.5,1438.3) \\
4 \mathrm{h:} 1067.0 \mathrm{\mu g} / \mathrm{kg} \\
(840.4,1356.1)\end{array}$ & $\begin{array}{l}\text { Potency ratio at } 2 \text { h: } 3.7 \\
\text { Potency ratio at } 4 \text { h: } 5.8\end{array}$ & \\
\hline & & Buprenorphine t.d. & Delta-9-THC (400 mg/kg t.d.) & $\begin{array}{l}2 \mathrm{h:} 4.3 \mathrm{mg} / \mathrm{kg}(2.8,6.8) \\
4 \mathrm{h:} 2.2 \mathrm{mg} / \mathrm{kg}(1.1,4.6)\end{array}$ & $\begin{array}{l}2 \mathrm{h:}: 26.1 \mathrm{mg} / \mathrm{kg}(17.1,39.9) \\
4 \mathrm{h:}: 15.6 \mathrm{mg} / \mathrm{kg}(10.0,24.5)\end{array}$ & $\begin{array}{l}\text { Potency ratio at } 2 \mathrm{h:}: 8.2 \\
\text { Potency ratio at } 4 \mathrm{h:} 7.2\end{array}$ & \\
\hline Cox et al, 2007 & $\begin{array}{l}\text { Paw pressure test (Male } \\
\text { Sprague-Dawley rats) }\end{array}$ & $\begin{array}{l}\text { Morphine i.p. (normal rats) } \\
\text { Morphine i.p. (arthritic rats) }\end{array}$ & $\begin{array}{l}\text { Delta-9-THC } \\
\text { (0.4 mg/kg } \pm 0.5 \text { i.p.) } \\
\text { ( } 1: \text { I ratio THC: Morphine) } \\
\text { Delta-9-THC (0.6 mg/ } \\
\text { kg } \pm 0.55 \text { i.p.) } \\
\text { ( } 1: \text { I ratio THC: Morphine) }\end{array}$ & $\begin{array}{l}0.4 \mathrm{mg} / \mathrm{kg} \pm 0.5 \\
0.6 \mathrm{mg} / \mathrm{kg} \pm 0.55\end{array}$ & $\begin{array}{l}2.4 \mathrm{mg} / \mathrm{kg}(2.2,2.8) \\
2.2 \mathrm{mg} / \mathrm{kg}(1.9,2.4)\end{array}$ & $\begin{array}{l}\text { The combination of delta-9-THC } \\
\text { and morphine showed synergism in } \\
\text { both non-arthritic and arthritic rats }\end{array}$ & $\begin{array}{l}\text { Results from normal rats included in } \\
\text { the meta-analysis only }\end{array}$ \\
\hline Finn et al, 2004 & $\begin{array}{l}\text { Formalin-evoked } \\
\text { nociceptive behavior } \\
\text { (adult male Lister- } \\
\text { Hooded rats) }\end{array}$ & Morphine i.p. & Delta-9-THC (I mg/kg i.p.) & Not reported & Not reported & $\begin{array}{l}\text { Not clearly synergistic. Potentially } \\
\text { additive. Morphine }(2 \mathrm{mg} / \mathrm{kg})+ \\
\text { delta-9-THC }(1 \mathrm{mg} / \mathrm{kg}) \mathrm{had} \text { a } \\
\text { significant effect on nociceptive } \\
\text { behavior (compared to morphine } \\
\text { alone but not delta-9-THC alone). }\end{array}$ & \\
\hline
\end{tabular}


Table I Continued

Equipotent opioid dose represented as $\mathrm{ED}_{50}(95 \% \mathrm{CL})$ or $\pm \mathrm{SEM}$, unless measured otherwise specified

\begin{tabular}{|c|c|c|c|c|c|c|c|}
\hline $\begin{array}{l}\text { Study } \\
\text { reference }\end{array}$ & Pain model (species) & Opioid administered & $\begin{array}{l}\text { Cannabinoid } \\
\text { administered }\end{array}$ & Cannabinoid condition & Vehicle condition & $\begin{array}{l}\text { Potency ratio or evidence of } \\
\text { synergism }\end{array}$ & Other notes \\
\hline $\begin{array}{l}\text { Katsuyama } \\
\text { et al, } 2013\end{array}$ & $\begin{array}{l}\text { Capsaicin test (Male mice } \\
\text { of ddY strain) }\end{array}$ & $\begin{array}{l}\text { Morphine ( } 1.0 \mathrm{mg} / \mathrm{kg} \text { s.c. and } \\
100 \text { pmol i.t.) }\end{array}$ & $\begin{array}{l}\text { Beta-caryophyllene }(2.25 \mathrm{mg} \\
\text { i.pl., CB2 receptor agonist) }\end{array}$ & $\begin{array}{l}1 D_{50} 1.16 \mathrm{mg} / \mathrm{kg} \\
(1.03,1.32 \text {, systemic, s.c.) } \\
\text { and I } 30.1 \text { pmol (I I I.9, } \\
\text { I56.4, spinal, i.t.) }\end{array}$ & $\begin{array}{l}\text { ID } D_{50} 2.5 I \mathrm{mg} / \mathrm{kg}(2.17,2.97) \\
\text { (systemic, s.c.) and } \\
193.7 \mathrm{pmol}(165.7,225.6, \\
\text { spinal, i.t.) }\end{array}$ & $\begin{array}{l}\text { Morphine }+ \text { beta-caryophyllene } \\
\text { decreased licking/biting response } \\
p<0.05 \text { compared to morphine + } \\
\text { saline or beta-caryophyllene + } \\
\text { jojoba wax. }\end{array}$ & $\begin{array}{l}\text { Ineffective doses of beta- } \\
\text { caryophyllene significantly } \\
\text { enhanced morphine-induced } \\
\text { antinociception. }\end{array}$ \\
\hline Li et al, 2008 & $\begin{array}{l}\text { Thermal antinociception } \\
\text { (mesus monkeys) }\end{array}$ & Morphine s.c. & $\begin{array}{l}\text { Delta-9-THC ( } 0.32 \text { and } \\
1.0 \mathrm{mg} / \mathrm{kg} \text { s.c.) }\end{array}$ & $\mathrm{ED}_{80} 2.42 \mathrm{mg} / \mathrm{kg}$ & $\mathrm{ED}_{80} 6.36 \mathrm{mg} / \mathrm{kg}(3.8 \mathrm{l}, 8.91)$ & $\begin{array}{l}\text { Pre-treatment with delta-9-THC } \\
\text { enhanced the antinociceptive } \\
\text { effects of morphine. }\end{array}$ & $\begin{array}{l}\text { Morphine dose dependently } \\
\text { increased the latency for monkeys } \\
\text { to remove their tails from } 50^{\circ} \mathrm{C} \text { and } \\
55^{\circ} \mathrm{C} \text { water. }\end{array}$ \\
\hline $\begin{array}{l}\text { Maguire et al, } \\
2013\end{array}$ & $\begin{array}{l}\text { Warm water tail } \\
\text { withdrawal (rhesus } \\
\text { monkeys) }\end{array}$ & Morphine s.c. & $\begin{array}{l}\text { CP } 55940(0.01 \mathrm{mg} / \mathrm{kg} \text { s.c. }) \\
\text { WIN } 55212 \\
(0.32 \mathrm{mg} / \mathrm{kg} \text { s.c. })\end{array}$ & $\begin{array}{l}\text { Mean }(n=3) \\
\text { CP } 0.23 \mathrm{mg} / \mathrm{kg} \\
\text { WIN } 0.24 \mathrm{mg} / \mathrm{kg}\end{array}$ & $1.26 \mathrm{mg} / \mathrm{kg}($ mean, $n=3)$ & $\begin{array}{l}\text { Pre-treatment with CP } 55940 \\
\text { resulted in a mean leftward shift to } \\
\text { of }-6.73 \text {-fold. Pre-treatment with } \\
\text { WIN } 55212 \text { resulted in mean } \\
\text { leftward shift of - } 5.5 \text {-fold. }\end{array}$ & $\begin{array}{l}\text { Antinociception from the } \\
\text { combination appeared to be } \\
\text { achieved without an increase in } \\
\text { abuse liability. }\end{array}$ \\
\hline $\begin{array}{l}\text { Pugh et al, } \\
1996\end{array}$ & $\begin{array}{l}\text { Tail-fick test (mail ICR } \\
\text { mice) }\end{array}$ & Morphine i.t. & $\begin{array}{l}\text { Delta-9-THC ( } 6 \mu \mathrm{\mu cg} / \text { mouse } \\
\text { i.t., inactive analgesic dose })\end{array}$ & $0.01 \mathrm{mcg} / \mathrm{mouse}$ & $\begin{array}{l}0.318 \mathrm{mcg} / \mathrm{mouse}(2.825 \\
0.036)\end{array}$ & $\begin{array}{l}\text { Greater than additive effect } \\
\text { observed, clear leftward shift of } \\
\text { graph. }\end{array}$ & \\
\hline $\begin{array}{l}\text { Reche et al, } \\
1996\end{array}$ & $\begin{array}{l}\text { Tail-flick and hot plate } \\
\text { test (Swiss albino mice) }\end{array}$ & Morphine i.p. & Delta-9-THC i.p. & $\begin{array}{l}\text { NA } \\
\text { Only one dose of morphine } \\
(2 \mathrm{mg} / \mathrm{kg} \text { i.p.) examined. } \\
\text { Study measured change in } \\
\text { ED }_{50} \text { of delta-9-THC. }\end{array}$ & NA & $\begin{array}{l}\text { Morphine pre-administration } \\
\text { shifted the dose-response curve } \\
\text { for delta-9-THC to the left (a } 2.5- \\
\text { fold shiff for the tail-fick test and a } \\
\text { three-fold shift for the hot plate } \\
\text { test). Analgesic effect blocked by } \\
\text { SR-14I } 716 \text { (cannabinoid } \\
\text { antagonist) and naloxone. }\end{array}$ & \\
\hline \multirow[t]{2}{*}{$\begin{array}{l}\text { Smith et al, } \\
1998\end{array}$} & \multirow[t]{2}{*}{$\begin{array}{l}\text { Tail-flick and hot plate } \\
\text { test (male ICR mice) }\end{array}$} & Morphine s.c. & $\begin{array}{l}\text { Tail-flick: delta-9-THC } \\
\text { (4 mg/kg s.c.) }\end{array}$ & $\begin{array}{l}0.29 \mathrm{mg} / \mathrm{kg} \\
(95 \% \mathrm{Cl} 0.04,1.94)\end{array}$ & $2.81 \mathrm{mg} / \mathrm{kg}(2.24,3.53)$ & Potency ratio: 8.5 & \multirow{2}{*}{$\begin{array}{l}\text { Multiple conditions tested different } \\
\text { combinations of s.c and p.o } \\
\text { morphine. Only s.c. }+ \text { s.c. and p.o. } \\
\text { p.o. for the tail-flick test are } \\
\text { reported here. A paw withdrawal } \\
\text { test was also conducted to } \\
\text { demonstrate that enhancement of } \\
\text { antinociception was not limited to } \\
\text { the tail. }\end{array}$} \\
\hline & & Morphine p.o. & $\begin{array}{l}\text { Tail-flick: delta-9-THC } \\
(20 \text { mg/kg p.o.) }\end{array}$ & $2.8 \mathrm{mg} / \mathrm{kg}(2.0,3.9)$ & $31.7 \mathrm{mg} / \mathrm{kg}(22.4,44.9)$ & Potency ratio: 7.6 & \\
\hline $\begin{array}{l}\text { Smith et al, } \\
2007\end{array}$ & $\begin{array}{l}\text { Paw withdrawal test } \\
\text { (male Sprague-Dawley } \\
\text { rats) }\end{array}$ & Morphine s.c. & $\begin{array}{l}\text { Delta-9-THC } \\
\text { (0.75 mg / kg i.p.) }\end{array}$ & $\begin{array}{l}\text { ED } 80 \text { morphine }+ \\
\text { delta-9-THC }(0.75 \mathrm{mg} / \mathrm{kg})\end{array}$ & $\begin{array}{l}\text { ED } 80 \text { morphine alone } \\
(100 \mathrm{mg} / \mathrm{kg})\end{array}$ & $\begin{array}{l}\text { Tolerance to morphine alone } \\
\text { rapidly established; no loss of effect } \\
\text { with low-dose combinations of } \\
\text { morphine + delta-9-THC }\end{array}$ & $\begin{array}{l}\text { A morphine pellet arm and delta-9- } \\
\text { THC alone arm were not reported } \\
\text { in this table due to difficulties in } \\
\text { comparing doses between } \\
\text { morphine formulations. }\end{array}$ \\
\hline $\begin{array}{l}\text { Tham et al, } \\
2005\end{array}$ & $\begin{array}{l}\text { Tail-flick and hot plate } \\
\text { test (Swiss male mice) }\end{array}$ & Morphine s.c. & $\begin{array}{l}\text { Tail-fick: CP } 55940 \\
\text { (0.1-3 mg/kg s.c.) } \\
\text { Hot plate: CP } 55940 \\
\text { (0.1-3 mg/kg s.c.) }\end{array}$ & $\begin{array}{l}3.31 \mathrm{mg} / \mathrm{kg} \\
7.54 \mathrm{mg} / \mathrm{kg}\end{array}$ & $\begin{array}{l}11.3 \mathrm{mg} / \mathrm{kg}(9.6,13.4) \\
29.4 \mathrm{mg} / \mathrm{kg}(27.3,31.6)\end{array}$ & $\begin{array}{l}\text { Analyses showed greater than } \\
\text { additive results (synergism). }\end{array}$ & \\
\hline $\begin{array}{l}\text { Wakley and } \\
\text { Craft, 2011 }\end{array}$ & $\begin{array}{l}\text { Paw pressure test (male } \\
\text { Sprague-Dawley rats) }\end{array}$ & Methadone i.p. & $\begin{array}{l}\text { Delta-9-THC } \\
(0.32-3.2 \mathrm{mg} / \mathrm{kg} \text { i.p. })\end{array}$ & $\begin{array}{l}\text { Not reported (dose- } \\
\text { response curve shown) }\end{array}$ & $\begin{array}{l}\mathrm{ED}_{50} \text { in naive rats, } 1.27 \mathrm{mg} / \mathrm{kg} \\
(95 \% \% \mathrm{Cl} 0.91,1.91) \text {, ED } 50 \text { in } \\
\text { rats trained for discrimination, } \\
3.49 \mathrm{mg} / \mathrm{kg}(95 \% \mathrm{Cl} 2.59 \text {, } \\
5.31)\end{array}$ & $\begin{array}{l}\text { In opioid and delta-9-THC naive } \\
\text { rats, methadone } 1.0 \mathrm{mg} / \mathrm{kg} \\
\text { significantly enhanced the } \\
\text { antinociceptive effect of delta-9- } \\
\text { THC, however this was not } \\
\text { observed in rats that were } \\
\text { previously trained for drug } \\
\text { discrimination tasks. }\end{array}$ & $\begin{array}{l}\text { The rats trained for drug } \\
\text { discrimination tasks had received } \\
\text { repeated administration of opioids } \\
\text { and cannabinoids over many } \\
\text { months and may have been } \\
\text { tolerant to drug effects at the doses } \\
\text { administered. }\end{array}$ \\
\hline \multirow[t]{2}{*}{$\begin{array}{l}\text { Welch and } \\
\text { Stevens, } 1992\end{array}$} & \multirow[t]{2}{*}{$\begin{array}{l}\text { Tail-flick and hot plate } \\
\text { test (mice) }\end{array}$} & \multirow[t]{2}{*}{ Morphine i.t. } & $\begin{array}{l}\text { Delta-9-THC } \\
\text { (3.133 mcg/mouse) }\end{array}$ & $\begin{array}{l}0.15 \mathrm{mcg} / \mathrm{mouse} \\
(0.11,0.21)\end{array}$ & \multirow[t]{2}{*}{$0.61 \mathrm{mcg} / \mathrm{mouse}(0.26,1.44)$} & Yes & \\
\hline & & & $\begin{array}{l}\text { Delta-9-THC } \\
\text { ( } 6.25 \mathrm{mcg} / \text { mouse })\end{array}$ & $\begin{array}{l}0.05 \mathrm{mcg} / \mathrm{mouse} \\
(0.03,0.08)\end{array}$ & & Yes & \\
\hline
\end{tabular}


Table I Continued

Equipotent opioid dose represented as $\mathrm{ED}_{50}(95 \% \mathrm{CL})$ or $\pm \mathrm{SEM}$, unless measured otherwise specified

\begin{tabular}{|c|c|c|c|c|c|c|c|}
\hline $\begin{array}{l}\text { Study } \\
\text { reference }\end{array}$ & Pain model (species) & Opioid administered & $\begin{array}{l}\text { Cannabinoid } \\
\text { administered }\end{array}$ & Cannabinoid condition & Vehicle condition & $\begin{array}{l}\text { Potency ratio or evidence of } \\
\text { synergism }\end{array}$ & Other notes \\
\hline & & & $\begin{array}{l}\text { Delta-8-THC } \\
\text { (25 mcg/mouse) }\end{array}$ & $\begin{array}{l}0.05 \mathrm{mcg} / \mathrm{mouse} \\
(0.02,0.10)\end{array}$ & & Yes & \\
\hline & & & $\begin{array}{l}\text { Levonantradol } \\
\text { (0.005 mcg/mouse) }\end{array}$ & $\begin{array}{l}0.06 \mathrm{mcg} / \mathrm{mouse} \\
(0.01,0.24)\end{array}$ & & Yes & \\
\hline & & & CP 55940 (0.01 mcg/mouse) & $0.3 \mathrm{mcg} / \mathrm{mouse}(0.0,0.10)$ & & Additive & \\
\hline & & & CP 56667 (0.5 mcg/mouse) & $\begin{array}{l}0.26 \mathrm{mcg} / \mathrm{mouse} \\
(0.08,0.82)\end{array}$ & & Additive & \\
\hline & & & $\begin{array}{l}\text { 11-hydroxy-delta-9-THC } \\
\text { (3 mcg/mouse) }\end{array}$ & $\begin{array}{l}0.08 \mathrm{mcg} / \mathrm{mouse} \\
(0.04,0.19)\end{array}$ & & Yes & \\
\hline & & & $\begin{array}{l}\text { Dextronantradol } \\
\text { ( } 25 \mathrm{mcg} / \text { mouse) }\end{array}$ & $\begin{array}{l}0.51 \mathrm{mcg} / \mathrm{mouse} \\
(0.36,0.89)\end{array}$ & & No & \\
\hline $\begin{array}{l}\text { Williams et al, } \\
2006\end{array}$ & $\begin{array}{l}\text { Tail-flick test } \\
\text { (mail ICR mice) }\end{array}$ & $\begin{array}{l}\text { Study I: low-dose codeine } \\
(30 \mathrm{mg} / \mathrm{kg}) \text { and morphine } \\
(20 \mathrm{mg} / \mathrm{kg}) \text { and fully efficacious } E_{80}, \\
\text { codeine (100 mg/kg) and morphine } \\
(80 \mathrm{mg} / \mathrm{kg}) \text {. Study 2: high-dose } \\
\text { codeine (200 mg/kg) and morphine } \\
(100 \mathrm{mg} / \mathrm{kg}) \text { (all p.o.) }\end{array}$ & $\begin{array}{l}\text { Delta-9-THC (20 mg/kg p.o., } \\
\text { inactive analgesic dose) }\end{array}$ & $\begin{array}{l}E D_{80} \text { codeine }(30 \mathrm{mg} / \mathrm{kg}) \\
\text { ED } 80 \text { morphine }(20 \mathrm{mg} / \mathrm{kg})\end{array}$ & $\begin{array}{l}E D_{80} \text { codeine }(200 \mathrm{mg} / \mathrm{kg}) \\
\text { ED } 80 \text { morphine }(100 \mathrm{mg} / \mathrm{kg})\end{array}$ & $\begin{array}{l}\text { A low dose of morphine }(20 \mathrm{mg} / \\
\mathrm{kg}) \text { or codeine }(30 \mathrm{mg} / \mathrm{kg}) \text { with a } \\
\text { single pre-treatment of an inactive } \\
\text { dose of delta-9-THC produced the } \\
\text { same efficacy (ED } \mathrm{ED} \text { ) as the high } \\
\text { doses of each opioid alone. For } \\
\text { codeine, delta-9-THC pre- } \\
\text { treatment also increased the } \\
\text { duration of action of the } \mathrm{ED}_{80} \text { dose } \\
\text { of codeine. }\end{array}$ & $\begin{array}{l}\text { Study I: pre-treatment with delta- } \\
\text { 9-THC did not enhance the fully } \\
\text { efficacious dose of morphine but } \\
\text { enhanced low-dose morphine and } \\
\text { both doses of codeine, in addition } \\
\text { to extending the time course. Study } \\
\text { 2: delta-9-THC restored analgesic } \\
\text { efficacy after the time that the } \\
\text { opioids had ceased being effective } \\
\text { on their own ( } 360 \text { min post dose } \\
\text { for morphine and } 120 \text { min post } \\
\text { dose for codeine). }\end{array}$ \\
\hline $\begin{array}{l}\text { Williams et al, } \\
2008\end{array}$ & $\begin{array}{l}\text { Tail-flick test (diabetic } \\
\text { and non-diabetic mice } \\
\text { and rats) }\end{array}$ & $\begin{array}{l}\text { Morphine s.c. } \\
\text { Morphine s.c. }\end{array}$ & $\begin{array}{l}\text { Delta-9-THC (20 mg/kg p.o.) } \\
\text { in non-diabetic mice } \\
\text { Delta-9-THC (20 mg/kg p.o.) } \\
\text { in diabetic mice }\end{array}$ & $\begin{array}{l}2.5 \mathrm{mg} / \mathrm{kg}(1.8,3.4) \\
0.84 \mathrm{mg} / \mathrm{kg}(0.79,0.89)\end{array}$ & $\begin{array}{l}5.6 \mathrm{mg} / \mathrm{kg}(4.3,7.2) \\
6.1 \mathrm{mg} / \mathrm{kg}(5.2,7.1)\end{array}$ & $\begin{array}{l}\text { Yes } \\
\text { Yes }\end{array}$ & $\begin{array}{l}\text { Delta-9-THC significantly enhanced } \\
\text { morphine-induced antinociception } \\
\text { in both diabetic and non-diabetic } \\
\text { mice. }\end{array}$ \\
\hline $\begin{array}{l}\text { Wilson et al, } \\
2008\end{array}$ & $\begin{array}{l}\text { Hot plate test (male } \\
\text { Sprague-Dawley rats) }\end{array}$ & $\begin{array}{l}\text { Morphine microinjections } \\
\text { into PAG }\end{array}$ & $\mathrm{HU}-2 \mathrm{IO}(5 \mu \mathrm{g})$ & $\begin{array}{l}\text { Not reported (dose- } \\
\text { response curve shown) }\end{array}$ & $\begin{array}{l}\text { Not reported (dose- } \\
\text { response curve shown) }\end{array}$ & $\begin{array}{l}\text { No evidence of synergism. } \\
\text { Morphine + HU- } 10 \text { showed the } \\
\text { greatest increase in hot plate } \\
\text { latency }(39.9 \mathrm{~s} \pm 1.1 \mathrm{~s}) \text {, but was not } \\
\text { significantly different from } \\
\text { morphine alone }(33.1 \mathrm{~s} \pm 4.0 \mathrm{~s})\end{array}$ & $\begin{array}{l}\text { HU-210 shown to prevent } \\
\text { development of tolerance to } \\
\text { morphine's antinociceptive effects. } \\
\text { HU-2 } 10 \text { pre-treatment enhanced } \\
\text { subsequent morphine } \\
\text { antinociception. Co-administration } \\
\text { of HU-210 into the PAG } \\
\text { attenuated morphine } \\
\text { antinociception. The authors } \\
\text { suggested that opioids and } \\
\text { cannabinoids may have opposing } \\
\text { actions within the PAG. }\end{array}$ \\
\hline $\begin{array}{l}\text { Yesilyurt et al, } \\
2003\end{array}$ & $\begin{array}{l}\text { Tail-flick test (adult } \\
\text { female Bulb-C mice) }\end{array}$ & Morphine topical & $\begin{array}{l}\text { WIN 55, 212-2 (20 mg/ml, } \\
\text { topical, mixed CBI-CB2 } \\
\text { receptor agonist) }\end{array}$ & $\begin{array}{l}\text { Morphine }(20 \mathrm{mg} / \mathrm{ml})+ \\
\text { WIN sustained analgesic } \\
\text { effect of } 50 \% \text { analgesia } \\
\text { over } 4 \mathrm{~h}\end{array}$ & $\begin{array}{l}\text { Morphine }(20 \mathrm{mg} / \mathrm{ml}) \text { alone } \\
\text { produced } 18 \% \text { analgesic } \\
\text { effect, peak at } 20 \mathrm{~min} \text { then } \\
\text { reduced. }\end{array}$ & $\begin{array}{l}\text { Antinociceptive effects were } \\
\text { markedly potentiated (they peaked } \\
\text { and were sustained at } 30 \mathrm{~min} \text { ) } \\
\text { compared to morphine response } \\
\text { alone. }\end{array}$ & \\
\hline
\end{tabular}

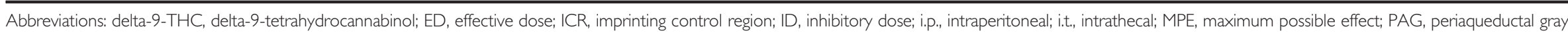
matter; pmol, picomol; p.o., oral administration; s.c., subcutaneous; t.d, transdermal. 


\section{Summary of Pre-Clinical Studies}

Nineteen pre-clinical studies were identified in which the analgesic effect of opioid and cannabinoid co-administration was examined (Cichewicz et al, 1999, 2005; Cichewicz and McCarthy, 2003; Cox et al, 2007; Finn et al, 2004; Katsuyama et al, 2013; Li et al, 2008; Maguire et al, 2013; Pugh Jr et al, 1996; Reche et al, 1996; Smith et al, 1998, 2007; Tham et al, 2005; Wakley and Craft, 2011; Welch and Stevens, 1992; Williams et al, 2006, 2008; Wilson et al, 2008; Yesilyurt et al, 2003) (Table 1). Fourteen of these studies examined delta-9THC, whereas one to two studies examined each of 10 other cannabinoid agonists, including beta-caryophyllene, CP 55 940, CP 56 667, delta-8-THC, 11-hydroxy-delta-9THC, dextronantradol, levonantradol, WIN 55, 212-2, and HU-210. Seventeen studies examined morphine, three studies examined codeine, and one to two studies examined buprenorphine, fentanyl, oxycodone, morphine, hydromorphone, methadone, LAAM, meperidine, and pentazocine. Most of the studies used rodents; however, two used rhesus monkeys and one used guinea pigs. The most common antinociceptive assays were tail-flick tests $(n=10)$ and hot plate tests $(n=5)$, although individual studies also used other forms of mechanical, thermal, and chemical nociception.

Most studies (17 of the 19) demonstrated that combining a cannabinoid with an opioid resulted in a synergistic effect on analgesia compared to the analgesic effects of the individual drugs. One study examined a single dose of morphine and demonstrated that morphine could potentiate the analgesic effect of intrathecally administered delta-9-THC (Reche et al, 1996). However, this study could not demonstrate an opioidsparing effect due to the use of a single dose of opioid. Another study found that $2 \mathrm{mg} / \mathrm{kg}$ morphine administered with $1 \mathrm{mg} / \mathrm{kg}$ delta-9-THC resulted in a significant effect on nociception compared to morphine alone $(p<005)$, but not when compared to delta-9-THC alone (Finn et al, 2004). In another study, a greater increase in hot plate latency was found for morphine combined with HU-210 (38.9s $\pm 1.1 \mathrm{~s})$ compared with HU-210 alone (33.1 s $\pm 4.0 \mathrm{~s})$ (Wilson et al, 2008); however, this difference did not reach significance.

One study testing multiple opioid agonists identified clear synergistic effects for delta-9-THC for most opioid drugs, with the exception of fentanyl and pentazocine (Cichewicz et al, 1999). The potency ratio when administered alone for those opioids found to have a synergistic effect, compared to when those same opioids were co-administered with delta-9THC varied between 2.2 and 25.8. Another study tested multiple cannabinoid agonists when co-administered with morphine and demonstrated a synergistic effect with delta-9THC, delta-8-THC, levonantradol, and 11-hydroxy-delta-9THC; additive effects with CP 55940 and CP 56 667; and no observable potentiation of morphine effects with dextronantradol, which is an isomer of levonantradol (Welch and Stevens, 1992). In contrast to the finding of an additive effect for CP 55940, two other studies of CP 55940 in combinations with morphine demonstrated a synergistic analgesic effect (Maguire et al, 2013; Tham et al, 2005). In addition to changes in the magnitude of the analgesic effect, two studies showed that the duration of the analgesic effect can be extended by administrating a low-dose opioid and cannabinoid in combination, compared with administrating an opioid alone (Williams et al, 2006; Yesilyurt et al, 2003).

\section{Meta-Analysis of Pre-Clinical Studies}

Six studies used sufficiently similar approaches to enable a meta-analysis (Cichewicz et al, 1999; Cichewicz and Welch, 2003; Cox et al, 2007; Smith et al, 1998; Welch and Stevens, 1992; Williams et al, 2008) (Figure 2). A further four studies were comparable in study design, but did not contain the required data $\left(\mathrm{ED}_{50}\right.$ or variance on estimates) to enable meta-analysis (Finn et al, 2004; Pugh Jr et al, 1996; Smith et al, 2007; Williams et al, 2006). All studies included in the meta-analysis used rodents and reported comparable antinociceptive doses of morphine alone and morphine coadministered with delta-9-THC. Results from the metaanalysis are reported in terms of mean difference.

The meta-analysis identified a significant opioid-sparing effect with morphine and delta-9-THC co-administration, $Z=5.59, p<0.001$ (MD in $\log _{10} \mathrm{ED}_{50}-0.56(-0.83,-0.29)$ ). As there was significant heterogeneity in the data $\left(I^{2}=95 \%\right)$, a random effects model was used. When back-transformed to the original units, the response ratio was 3.6 (95\% CI 1.95, 6.76), indicating that the median $\mathrm{ED}_{50}$ of morphine was 3.6 times lower when given in combination with delta9-THC compared to when morphine was administered alone.

Two studies compared doses of codeine with and without delta-9-THC in rodents (Cichewicz et al, 1999; Cichewicz and Welch, 2003) (Figure 3). Both studies used male ICR mice and the tail-flick assay. Meta-analysis of these data indicated a significant opioid-sparing effect of delta-9THC when co-administered with codeine, $Z=2.49, p=0.01$

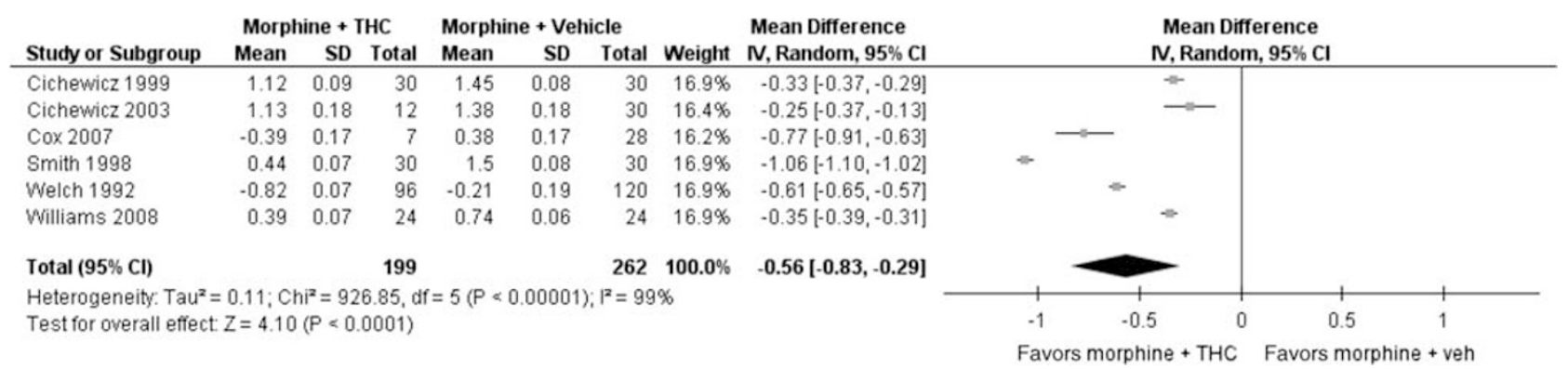

Figure 2 Forrest plot for meta-analysis examining the opioid-sparing effect of delta-9-THC when co-administered with morphine. Note: all mean difference and $\mathrm{SD}$ values are of $\log _{10} \mathrm{ED}_{50}$. THC, tetrahydrocannabinol. 


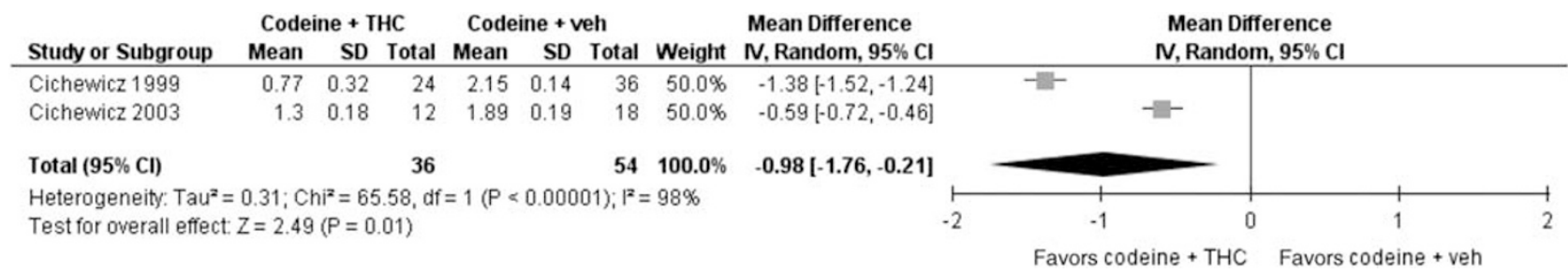

Figure 3 Forrest plot for meta-analysis examining the opioid-sparing effect of delta-9-THC when co-administered with codeine. Note: all mean difference and $\mathrm{SD}$ values are of $\log _{10} \mathrm{ED}_{50}$. THC, tetrahydrocannabinol.

(MD in the $\log _{10} \mathrm{ED}_{50}-0.98(-1.76,-0.21)$ ). Significant heterogeneity in the data $\left(I^{2}=98 \%\right)$ necessitated the use of a random effects model. When back-transformed to the original units, the response ratio was 9.5 (95\% CI 1.6, 57.5), indicating that the $\mathrm{ED}_{50}$ of codeine was 9.5 times lower when given in combination with delta-9-THC compared to when codeine was administered alone.

Funnel plots did not provide evidence of publication or small study bias with these pre-clinical studies (Figure 4).

\section{Results from Clinical Studies}

Nine clinical studies with 750 participants provided data relevant to the research question (Table 2); however, the heterogeneous nature of the studies precluded meta-analysis. Three laboratory-based studies examined pain responses in participants concurrently being administered opioids and cannabinoids. One study recruited people with mixed chronic non-cancer pain $(n=24)$ who were prescribed opioids (Abrams et al, 2011). A significant reduction in pain ratings was observed for the participants in this study following co-administration of cannabinoids-39.6 (95\% CI $35.8,43.3)$ at baseline vs 29.1 (95\% CI 25.4, 32.8) following co-administration (Abrams et al, 2011). It should be noted that no placebo or control condition was used in this study for comparison (Abrams et al, 2011).

In another two studies, healthy volunteers $(n=12$ and 13 , respectively) participated in crossover studies, with single doses of placebo, morphine alone, dronabinol alone, and dronabinol and morphine combined administered over four sessions (Naef et al, 2003; Roberts et al, 2006). These studies did not identify a synergistic effect on experimental pain in healthy controls, although Roberts et al (2006) found that the co-administration of dronabinol and morphine resulted in a reduced unpleasantness of pain compared to either drug alone. In a case series examining the effects of cannabinoid administration in patients with chronic non-cancer pain, three patients with mixed pain conditions (multiple sclerosis, HIV-related peripheral neuropathy, and lower back and leg pain) reported reductions in opioid requirements after initiation of smoked cannabis plant material (Lynch and Clark, 2003).

Five controlled studies were identified. One small, nonrandomized study of patients with advanced cancer pain found that 5 out of 12 patients achieved pain control after receiving a cannabis infusion, compared with 2 out of 14 achieving pain control in the control group-a nonstatistically different effect (Lissoni et al, 2014). Two randomized controlled trials examined delta-9-THC: Cannabidiol (THC:CBD) combination oral sprays

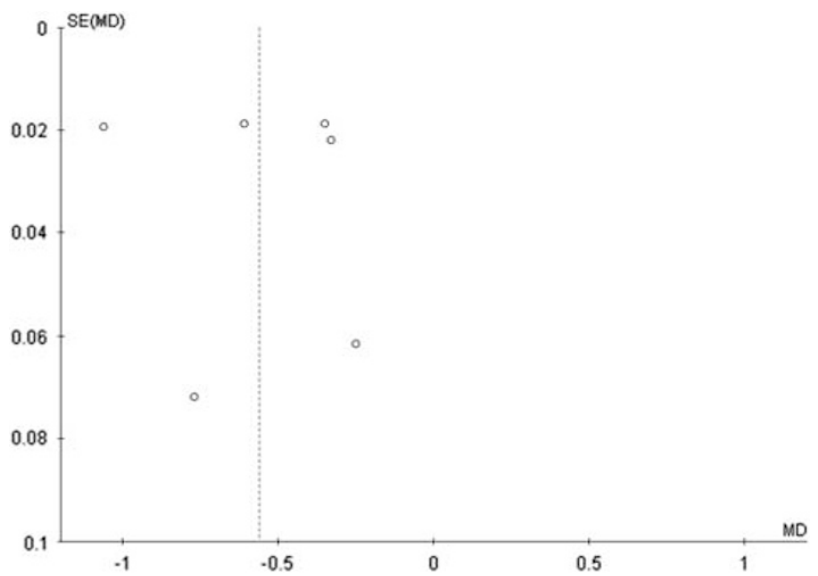

Figure 4 Funnel plot showing data from the six studies included in the meta-analysis. MD, mean difference, SE, standard error.

compared to a placebo (Johnson et al, 2010; Portenoy et al, 2012) in patients with cancer pain who were taking opioids. These studies found improved analgesia with the THC: CBD combination compared to the placebo. Johnson et al (2010) found no effect of THC: CBD on breakthrough opioid dose requirements. Portenoy et al (2012) conducted a doseranging study, using fixed dose ranges of the THC:CBD combination. In this study, a significant analgesic effect was only found in the lowest dose group, with poorer tolerability observed for higher doses.

Two controlled studies examined the effects of dronabinol: one in patients with mixed chronic pain (Narang et al, 2008) and one in patients with prostate cancer (Seeling et al, 2006). Narang et al (2008) found significantly reduced pain intensity with the opioid-cannabinoid combination in double-blinded laboratory sessions compared to opioid alone. Additional improvements in sleep, energy, and social functioning were reported in a 4-week open-label phase of the same study (Narang et al, 2008). In the study by Seeling et al (2006), perioperative use of dronabinol compared with a placebo in patients with prostate cancer, no difference was found in self-administered opioid dose requirements between groups.

\section{Quality Ratings of Clinical Studies}

The clinical studies were rated using the GRADE criteria. One study provided very-low-quality evidence, three studies provided low-quality evidence, two studies provided moderate-quality evidence, and three randomized controlled 
Table 2 Summary of Evidence of Opioid-sparing Effects from Clinical Studies

\begin{tabular}{|c|c|c|c|c|c|c|c|c|c|c|}
\hline $\begin{array}{l}\text { (a) Study } \\
\text { reference }\end{array}$ & Study design & Population & $\begin{array}{l}\text { Follow-up } \\
\text { period }\end{array}$ & Opioid used & Canr & nabinoid used & \multicolumn{2}{|c|}{$\begin{array}{l}\text { Effect of cannabinoid on } \\
\text { opioid dose }\end{array}$} & $\begin{array}{l}\text { Outcome on analgesia } \\
\text { observed }\end{array}$ & $\begin{array}{l}\text { GRADE evidence rating } \\
\text { and other notes }\end{array}$ \\
\hline \multicolumn{11}{|c|}{ Laboratory studies } \\
\hline $\begin{array}{l}\text { Abrams } \\
\text { et al, 201। }\end{array}$ & $\begin{array}{l}\text { Clinical laboratory study of } \\
\text { self-reported pain under } \\
\text { observed conditions (also } \\
\text { measured pharmacokinetic } \\
\text { effects of concurrent } \\
\text { administration) }\end{array}$ & $\begin{array}{l}\text { People }(n=24) \\
\text { receiving chronic } \\
\text { opioid treatment } \\
\text { (mixed pain } \\
\text { conditions) }\end{array}$ & 5 days & $\begin{array}{l}\text { Morphine sulfate } \\
\text { (mean daily dose } \\
62 \mathrm{mg}, n=13 \text { ) or } \\
\text { oxycodone } \\
\text { hydrochloride (mean } \\
\text { daily dose } 53 \mathrm{mg} \text {, } \\
n=11 \text { ) }\end{array}$ & $\begin{array}{l}\text { Vapor } \\
\text { of } 0.9 \\
9 \text {-TH } \\
\text { they } \\
\text { admin } \\
\text { per d }\end{array}$ & $\begin{array}{l}\text { rized cannabis dose } \\
\text { g of } 3.56 \% \text { delta- } \\
\text { C or as much as } \\
\text { could tolerate, } \\
\text { histered three times } \\
\text { ay. }\end{array}$ & \multicolumn{2}{|c|}{$\begin{array}{l}\text { Opioid dose held constant to } \\
\text { examine effect of delta-9- } \\
\text { THC on opioid } \\
\text { pharmacokinetics (ie, no } \\
\text { reduction from baseline } \\
\text { opioid dose possible). }\end{array}$} & $\begin{array}{l}\text { Mean pain score reduction, } \\
\text { from } 34.8(95 \% \mathrm{Cl} 29.4,40.1) \\
\text { at baseline to } 24.1(95 \% \mathrm{Cl} \\
\text { 18.8, } 29.4) \text { on day } 5 \text { with } \\
\text { morphine, and from } 43.8 \\
(95 \% \mathrm{Cl} 38.6,49.1) \text { at baseline } \\
\text { to } 33.6(95 \% \mathrm{Cl} 28.5,38.6) \text { on } \\
\text { day } 5 \text { with oxycodone. } \\
\text { Significant reduction overall. }\end{array}$ & $\begin{array}{l}\text { Cannabis inhalation } \\
\text { produced a subjective 'high'. } \\
\text { GRADE rating 'low' quality. } \\
\text { Downgraded as study did } \\
\text { not have a placebo } \\
\text { condition, so placebo effects } \\
\text { cannot be excluded } \\
\text { Note: no pharmacokinetic } \\
\text { interaction observed. }\end{array}$ \\
\hline $\begin{array}{l}\text { Naef et al, } \\
2003\end{array}$ & $\begin{array}{l}\text { Experimental heat, cold, } \\
\text { pressure, single and repeatec } \\
\text { transcutaneous electrical } \\
\text { stimulation pain, } \\
\text { randomized, placebo- } \\
\text { controlled, double-blinded, } \\
\text { crossover study. }\end{array}$ & $\begin{array}{l}\text { Healthy cannabis } \\
\text { naive volunteers } \\
(n=12)\end{array}$ & $\begin{array}{l}\text { Four study } \\
\text { sessions with at } \\
\text { least } 7 \text { days } \\
\text { washout } \\
\text { between sessions }\end{array}$ & $\begin{array}{l}\text { Morphine (30 mg) } \\
\text { daily }\end{array}$ & Dron & abinol (20 mg) & \multicolumn{2}{|c|}{$\begin{array}{l}\text { No significant analgesic effect } \\
\text { of dronabinol or morphine-- } \\
\text { dronabinol combination on } \\
\text { heat, pressure, or cold tests. } \\
\text { Additive effect of morphine } \\
\text { on transcutaneous electrical } \\
\text { stimulation test. }\end{array}$} & $\begin{array}{l}\text { Potentiation of analgesia not } \\
\text { observed in this experimental } \\
\text { pain study. }\end{array}$ & $\begin{array}{l}\text { GRADE rating 'moderate'. } \\
\text { Placebo-controlled, blinded } \\
\text { study. Downgraded due to } \\
\text { indirect evidence as use of } \\
\text { experimental pain measures. }\end{array}$ \\
\hline $\begin{array}{l}\text { Roberts } \\
\text { et al, } 2006\end{array}$ & \multicolumn{2}{|c|}{$\begin{array}{ll}\text { Experimental thermal pain. } & \text { Healthy volunteers } \\
\text { Double-blinded, four } & (n=13) \text { with no } \\
\text { treatment crossover design. } & \begin{array}{l}\text { recent opioid or } \\
\text { cannabinoid use }\end{array}\end{array}$} & Four lab sessions & $\begin{array}{l}\text { Morphine }(0.02 \mathrm{mg} / \mathrm{kg} \\
\mathrm{N}, 1.4 \mathrm{mg} \text { dose for } \\
70 \mathrm{mg} \text { adult, ie, sub- } \\
\text { analgesic) }\end{array}$ & Dron & abinol (5 mg) & \multicolumn{2}{|c|}{$\begin{array}{l}\text { NA (opioid dose held } \\
\text { constant) }\end{array}$} & $\begin{array}{l}\text { Combination of delta-9-THC } \\
\text { and morphine did not have an } \\
\text { effect on pain intensity. The } \\
\text { combination resulted in lower } \\
\text { ratings of unpleasantness of } \\
\text { pain compared with either } \\
\text { drug alone. }\end{array}$ & $\begin{array}{l}\text { GRADE rating 'moderate'. } \\
\text { Placebo-controlled, blinded } \\
\text { study. Downgraded due to } \\
\text { indirect evidence as use of } \\
\text { experimental pain measures. }\end{array}$ \\
\hline \multicolumn{11}{|l|}{ Case series } \\
\hline $\begin{array}{l}\text { Lynch and } \\
\text { Clark, } \\
2003\end{array}$ & Observational case series & $\begin{array}{l}\text { Mixed pain } \\
\text { conditions }(n=3) \\
\text { (peripheral } \\
\text { neuropathy, } \\
\text { multiple sclerosis, } \\
\text { lower back pain) }\end{array}$ & $\begin{array}{l}\text { I-9-month } \\
\text { observation } \\
\text { period }\end{array}$ & $\begin{array}{l}\text { Morphine (75-360 mg } \\
\text { daily) }\end{array}$ & $\begin{array}{l}\text { Smok } \\
\text { unkno }\end{array}$ & $\begin{array}{l}\text { ed cannabis plant, } \\
\text { Dwn content }\end{array}$ & \multicolumn{2}{|c|}{$\begin{array}{l}\text { Mean baseline morphine dose } \\
\text { of } 195 \mathrm{mg} \text { (SD } 147 \mathrm{mg} \text { ) } \\
\text { compared with } 35 \mathrm{mg} \text { (SD } \\
31 \mathrm{mg} \text { ) after commencing } \\
\text { smoked cannabis. Opioid } \\
\text { dose reduction or cessation in } \\
\text { each case. }\end{array}$} & $\begin{array}{l}\text { Improved pain control } \\
\text { described, with patients either } \\
\text { reducing or ceasing morphine } \\
\text { dose. }\end{array}$ & GRADE rating 'very low'. \\
\hline $\begin{array}{l}\text { (b) Study } \\
\text { reference }\end{array}$ & Study design & pulation & $\begin{array}{l}\text { Follow-up } \\
\text { period }\end{array}$ & Opioid used & & Cannabinoid use & & $\begin{array}{l}\text { Effect of cannabinoid } \\
\text { on opioid dose }\end{array}$ & $\begin{array}{l}\text { Outcome on analgesia } \\
\text { observed }\end{array}$ & $\begin{array}{l}\text { GRADE evidence } \\
\text { rating and other notes }\end{array}$ \\
\hline \multicolumn{11}{|l|}{ Controlled trials } \\
\hline \multirow[t]{3}{*}{$\begin{array}{l}\text { Johnson } \\
\text { et al, } 2010\end{array}$} & \multirow[t]{3}{*}{$\begin{array}{l}\text { Multicenter, double- } \\
\text { blind, randomized, } \\
\text { placebo-controlled, } \\
\text { parallel-group trial. }\end{array}$} & \multirow[t]{3}{*}{$\begin{array}{l}\text { Patients with cancer pain } \\
(n=177) \text {, with inadequate } \\
\text { analgesia despite chronic } \\
\text { opioid dosing. }\end{array}$} & \multirow[t]{3}{*}{2 weeks } & $\begin{array}{l}\text { Varied opioids repo } \\
\text { OME (IQR) } \\
120 \text { mg (50-213) }\end{array}$ & & $\begin{array}{l}\text { Patients randomize } \\
\text { delta-9-THC: CBD } \\
\text { delta-9-THC, or } \\
\text { placebo } \\
\text { Delta-9-THC (mea } \\
\text { sprays per day) }\end{array}$ & $\begin{array}{l}\text { ed to } \\
\text { an } 9\end{array}$ & $\begin{array}{l}\text { No change in median } \\
\text { amount of breakthrough } \\
\text { opioid medication in any } \\
\text { group. }\end{array}$ & \multirow{2}{*}{$\begin{array}{l}\text { Change in pain NRS (out of } \\
\text { 10) from baseline was } \\
\text { statistically significantly in } \\
\text { favor of delta-9- } \\
\text { THC: CBD compared with } \\
\text { the placebo } \\
-1.01(p=0.245) \\
-1.37(p=0.014)\end{array}$} & \multirow[t]{3}{*}{$\begin{array}{l}\text { f GRADE rating 'high'. } \\
\text { Placebo-controlled and } \\
\text { randomized. }\end{array}$} \\
\hline & & & & 80 mg (30-180) & & \multicolumn{3}{|c|}{$\begin{array}{l}\text { Delta-9-THC: CBD } \\
\text { (mean } 10 \text { sprays } \\
\text { per day) }\end{array}$} & & \\
\hline & & & & 120 mg (40-240) & & \multicolumn{3}{|c|}{$\begin{array}{l}\text { Placebo } \\
\text { (mean II sprays/day) }\end{array}$} & -0.69 (reference group) & \\
\hline
\end{tabular}


Table 2 Continued

\begin{tabular}{|c|c|c|c|c|c|c|c|c|}
\hline $\begin{array}{l}\text { (b) Study } \\
\text { reference }\end{array}$ & Study design & Population & $\begin{array}{l}\text { Follow-up } \\
\text { period }\end{array}$ & Opioid used & Cannabinoid used & $\begin{array}{l}\text { Effect of cannabinoid } \\
\text { on opioid dose }\end{array}$ & $\begin{array}{l}\text { Outcome on analgesia } \\
\text { observed }\end{array}$ & $\begin{array}{l}\text { GRADE evidence } \\
\text { rating and other notes }\end{array}$ \\
\hline $\begin{array}{l}\text { Lissoni } \\
\text { et al, } 2014\end{array}$ & $\begin{array}{l}\text { Two groups (not } \\
\text { randomized): } \\
\text { cannabinoid tincture or } \\
\text { melatonin }\end{array}$ & $\begin{array}{l}\text { Patients with untreatable } \\
\text { metastatic solid tumor } \\
(n=26)\end{array}$ & Not stated & $\begin{array}{l}\text { Oxycodone, median dose } \\
\text { of } 30 \mathrm{mg}(10-60 \mathrm{mg}) \text {, } \\
\text { twice per day }\end{array}$ & $\begin{array}{l}\text { Cannabis flos ( } 19 \% \\
\text { delta- } 9-\mathrm{THC} \text { ) was given } \\
\text { as an infusion. } 100 \mathrm{ml} \\
(500 \mathrm{mg} / \mathrm{l} \text { water) three } \\
\text { times per day }\end{array}$ & $\begin{array}{l}5 / 12 \text { patients }(42 \%) \\
\text { achieved control of pain } \\
\text { without opioid dose } \\
\text { increase compared to the } \\
\text { control group, where } 2 / 14 \\
\text { (14\%) achieved pain } \\
\text { control }\end{array}$ & $\begin{array}{l}\text { The number that achieved } \\
\text { pain control was not } \\
\text { significantly different } \\
\text { between groups }\end{array}$ & $\begin{array}{l}\text { GRADE rating 'low'. Non- } \\
\text { randomized design, no } \\
\text { allocation concealment } \\
\text { described. Control group } \\
\text { received melatonin ( } 20- \\
100 \mathrm{mg} \text { ). Greater disease } \\
\text { progression documented } \\
\text { in the cannabis group }\end{array}$ \\
\hline \multirow[t]{2}{*}{$\begin{array}{l}\text { Narang } \\
\text { et al, } 2008\end{array}$} & $\begin{array}{l}\text { Phase I: randomized, } \\
\text { single-dose, double- } \\
\text { blinded, placebo- } \\
\text { controlled, crossover } \\
\text { trial. Primary outcome } \\
\text { measures TOTPAR } \\
\text { score }\end{array}$ & $\begin{array}{l}\text { Patients on opioids for } \\
\text { chronic pain; BPI } \geqslant 4 \\
(n=30) \text {. Pain diagnosis: } \\
\text { neuropathic }(n=7) \text {, } \\
\text { nociceptive }(n=7) \text {, mixed } \\
\text { neuropathic and } \\
\text { nociceptive }(n=\mid 1) \text {, and } \\
\text { uncategorized }(n=5)\end{array}$ & $\begin{array}{l}\text { Phase I: three } 8-\mathrm{h} \\
\text { lab sessions with } \\
3 \text { days washout }\end{array}$ & $\begin{array}{l}\text { OME mean } 68.1 \mathrm{mg}(\mathrm{SD} \\
57.2, \text { range } 7.5-228) . \\
\text { Participants were taking } \\
\text { oxycodone, morphine, } \\
\text { methadone hydrocodone, } \\
\text { and hydromorphone }\end{array}$ & $\begin{array}{l}\text { Phase I: single-dose } \\
\text { placebo, dronabinol } 10 \\
\text { and } 20 \mathrm{mg}\end{array}$ & $\begin{array}{l}\text { One subject took rescue } \\
\text { pain medication in all } \\
\text { conditions, one subject } \\
\text { took rescue medication } \\
\text { during the placebo and } \\
10 \mathrm{mg} \text { dronabinol } \\
\text { condition, and six subjects } \\
\text { took rescue medication } \\
\text { only with placebo. }\end{array}$ & $\begin{array}{l}\text { In single-dose studies, } 10 \\
\text { and } 20 \mathrm{mg} \text { dronabinol } \\
\text { significantly increased the } \\
\text { amount of analgesic relief } \\
\text { reported compared to } \\
\text { placebo }\end{array}$ & $\begin{array}{l}\text { GRADE rating 'moderate'. } \\
\text { Randomized and placebo- } \\
\text { controlled. Downgraded as } \\
\text { only a single dose was } \\
\text { examined. TOTPAR } 31.1 \\
\text { in placebo group, } \\
\text { compared with } 39.7 \text { with } \\
\text { dronabinol } 10 \mathrm{mg} \text { and } 41.7 \\
\text { with dronabinol } 20 \mathrm{mg}\end{array}$ \\
\hline & $\begin{array}{l}\text { Phase 2: open-label (no } \\
\text { placebo) extension. } \\
\text { Primary outcome } \\
\text { measure change in pain } \\
\text { intensity }\end{array}$ & & $\begin{array}{l}\text { Phase 2: open } \\
\text { label for } 4 \text { weeks }\end{array}$ & & $\begin{array}{l}\text { Phase 2: flexible dose } \\
\text { schedule, dronabinol } \\
5 \mathrm{mg} \text { daily - } 20 \mathrm{mg} \\
\text { three times per day. }\end{array}$ & Opioid dose not reported & $\begin{array}{l}\text { Mean baseline NRS of } 6.9 \\
\text { compared with } 5.2 \text { after } \\
4 \text { weeks of dronabinol. This } \\
\text { represents a statistically } \\
\text { significant reduction }\end{array}$ & $\begin{array}{l}\text { GRADE rating 'low'. Open- } \\
\text { label study. Significant } \\
\text { improvements }(p<0.05) \text { in } \\
\text { sleep, energy, pain relief, } \\
\text { and social functioning. Lack } \\
\text { of placebo control means } \\
\text { effects may be non-specific } \\
\text { or placebo }\end{array}$ \\
\hline $\begin{array}{l}\text { Portenoy } \\
\text { et al, } 2012\end{array}$ & $\begin{array}{l}\text { Randomized, 4-arm } \\
\text { placebo-controlled, } \\
\text { graded-dose study }\end{array}$ & $\begin{array}{l}\text { Patients with active cancer } \\
\text { and chronic pain on a } \\
\text { stable oral morphine } \\
\text { regimen, plus fentanyl } \\
(n=360)\end{array}$ & $\begin{array}{l}5 \text { weeks of } \\
\text { medication } \\
\text { administration }\end{array}$ & $\begin{array}{l}\text { Morphine and fentanyl } \\
\text { Median } 120 \mathrm{mg} \text { OME } \\
\text { Median } 120 \mathrm{mg} \text { OME } \\
\text { Median } 180 \mathrm{mg} \text { OME } \\
\text { Median } 120 \mathrm{mg} \text { OME }\end{array}$ & $\begin{array}{l}\text { Nabiximols } 1-4 \text { sprays } \\
\text { Nabiximols } 6-10 \text { sprays } \\
\text { Nabiximols } 11-16 \\
\text { sprays } \\
\text { Placebo }\end{array}$ & $\begin{array}{l}\text { No change in median } \\
\text { amount of breakthrough } \\
\text { opioid medication in any } \\
\text { group. Note that patients } \\
\text { were discouraged from } \\
\text { reducing their opioid dose, } \\
\text { so the opioid-sparing effect } \\
\text { could not be observed }\end{array}$ & $\begin{array}{l}\text { Treatment difference } \\
\text { (change from baseline pain } \\
\text { score): } \\
-0.75 \text { points ( } 95 \% \mathrm{Cl}-1.28 \text {, } \\
-0.22, p=0.06 \text { compared } \\
\text { to placebo) } \\
-0.36 \text { points }(95 \% \mathrm{Cl}-.089 \text {, } \\
0.18 \text { points, } p=0.19 \\
\text { compared to placebo). } \\
-0.09 \text { points ( } 95 \% \mathrm{Cl} \text { - } \\
0.62,0.44 \text { points, } p=0.75 \\
\text { compared to placebo) } \\
\text { Not reported } \\
\text { (reference group) }\end{array}$ & $\begin{array}{l}\text { GRADE rating 'high'. } \\
\text { Placebo-controlled, } \\
\text { randomized controlled trial } \\
\text { Opioid composite } \\
\text { measure showed better } \\
\text { improvements in the low- } \\
\text { dose group. I-4 spray } \\
\text { group had significant } \\
\text { improvements in analgesia. } \\
\text { Lower tolerability of delta- } \\
\text { 9-THC: CBD in higher } \\
\text { dose groups }\end{array}$ \\
\hline $\begin{array}{l}\text { Seeling } \\
\text { et al, } 2006\end{array}$ & $\begin{array}{l}\text { Randomized, } \\
\text { controlled trial (two } \\
\text { groups) }\end{array}$ & $\begin{array}{l}\text { Prostate cancer patients } \\
<70 \text { y.o. }(n=105) \text {. } \\
N=53 \text { in intervention and } \\
52 \text { in control }\end{array}$ & $\begin{array}{l}\text { From the day prior } \\
\text { to surgery to two } \\
\text { days post } \\
\text { operation }\end{array}$ & $\begin{array}{l}\text { Piritramide } 1.5 \mathrm{mg} / \mathrm{ml} \text {, } \\
\text { bolus } 2 \mathrm{mg} \text { (no continuous } \\
\text { infusion) via patient- } \\
\text { controlled analgesia for } \\
48 \mathrm{~h} \text { post operation }\end{array}$ & $\begin{array}{l}\text { Dronabinol } 5 \mathrm{mg} \times 8 \\
\text { doses over } 48 \mathrm{~h} \\
\text { (perioperatively) }\end{array}$ & $\begin{array}{l}\text { Median dose of piritramide } \\
\text { alone was } 74 \mathrm{mg} \text { (IQR } 44- \\
\text { 90) compared with } 54 \mathrm{mg} \\
\text { (IQR 46-88) when } \\
\text { administered with } \\
\text { dronabinol }\end{array}$ & $\begin{array}{l}\text { The difference between the } \\
\text { intervention (dronabinol) } \\
\text { group and control group } \\
\text { was not significant. No } \\
\text { evidence was found of } \\
\text { synergistic antinociceptive } \\
\text { interaction between delta- } \\
\text { 9-THC and piritramide for } \\
\text { acute postoperative pain }\end{array}$ & $\begin{array}{l}\text { GRADE rating 'high'. } \\
\text { Placebo-controlled, } \\
\text { randomized controlled trial } \\
\text { Patients administered their } \\
\text { own opioid doses }\end{array}$ \\
\hline
\end{tabular}

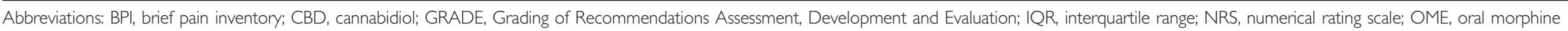
equivalents; TOTPAR, total pain relief. 
trials provided high-quality evidence. None of the highquality studies provided evidence of an opioid-sparing effect. The only study that provided direct evidence of an opioidsparing effect was rated as providing very low-quality evidence (Lynch and Clark, 2003).

\section{DISCUSSION}

Twenty-eight studies provided data relating to the potential opioid-sparing effect of cannabinoids in the context of opioid analgesia. Most of the pre-clinical studies examined reported reduced opioid requirements when co-administered with cannabinoids. Few controlled clinical studies measured opioid-sparing as an end point and findings relating to analgesia were mixed. Two controlled studies found no effect of cannabinoids on opioid dose requirements (Johnson et al, 2010; Seeling et al, 2006). One case series provided very low-quality evidence of a reduction in opioid dose requirements with cannabinoid co-administration (Lynch and Clark, 2003).

Most of the pre-clinical studies examined found synergistic effects when opioids and cannabinoids were co-administered, although two studies found that with specific opioids and cannabinoids the analgesic effect was additive rather than synergistic. Through meta-analyses, it was found that the doses of morphine and codeine required to produce the same analgesic effect were 3.6 and 9.5 times lower, respectively, when co-administered with delta-9-THC. Reductions in opioid requirements that are smaller than those seen in these pre-clinical studies may have relevance to pain treatment. Some confidence in these findings comes from the consistent observation of an opioid-sparing effect when using different nociceptive assays and in pain models of arthritis and diabetic neuropathy.

The relevance of the findings from these pre-clinical studies (with acute-dosing paradigms) to clinical chronic pain treatment must be considered. There are important limitations in translating findings from pre-clinical studies to clinical practice, particularly when evaluating doses and effect sizes. Although the outcomes of pre-clinical studies are often consistent with clinical studies, pre-clinical studies may over-represent effects. The lesser effect sizes in human studies have been attributed to the heterogeneity of clinical populations or the response being limited to sub-populations, reducing the overall effect observed (Berge, 2011). This underscores the importance of clinical studies to examine the effects found in pre-clinical work.

Controlled clinical studies demonstrated some beneficial effects of opioid and cannabinoid co-administration on outcomes of pain, sleep, and functioning in chronic pain patients (Abrams et al, 2011; Narang et al, 2008). One case series $(n=3)$ provided very low-quality evidence of a reduction in opioid requirements with delta-9-THC administration. No randomized controlled studies were identified that provided evidence of an opioid-sparing effect of cannabinoids. Important limitations identified in these clinical studies included a lack of placebo control (Abrams et al, 2011; Lynch and Clark, 2003; Narang et al, 2008), difficulties extrapolating from experimental to clinical pain (Naef et al, 2003; Roberts et al, 2006), use of single doses (Naef et al, 2003; Roberts et al, 2006), use of small sample sizes (Lissoni et al, 2014; Lynch and Clark, 2003; Narang et al, 2008), and the mixed quality of the study design in general. In particular, Roberts et al (2006) used sub-therapeutic doses of morphine, which may have limited that study's ability to test the effects of co-administration. Portenoy et al (2012) noted that the use of fixed dose ranges of cannabinoids may have limited that study's ability to test the efficacy of cannabinoids for pain, as some patients may have dropped out due to tolerability. Moreover, by discouraging patients from reducing their opioid dose during the study, no opioid-sparing effect could be observed (Portenoy et al, 2012).

This review highlights some important considerations for future studies of cannabinoids. A dose-ranging study with patients with advanced cancer found that only lower doses of cannabinoids demonstrated analgesic effects (Portenoy et al, 2012). In the same study, one in four participants in the high-dose group discontinued treatment. Side effects such as nausea, drowsiness, and dizziness are more frequent with higher doses of cannabinoids (Narang et al, 2008; Portenoy et al, 2012). This suggests that dose range and tolerability are important outcomes to examine and that careful dose titration is essential. Future studies should carefully document adverse effects from concurrent opioid and cannabinoid administration to provide a better understanding of potential harms. One hypothesis to explain why patients reduce their opioid dose with cannabinoid administration is that they experience undesirable psychoactive effects from concurrent use of opioids and cannabinoids. This could be explored in future studies.

Recent observational studies provided further data on a possible opioid-sparing effect. Two studies found $44-64 \%$ reductions in self-reported opioid consumption in cohorts of patients with chronic pain who were using cannabis (Boehnke et al, 2016; Haroutounian et al, 2016). These observational studies provide further low-quality evidence supporting an opioid-sparing effect. A further observational study found that in patients with chronic pain who were prescribed opioids, greater pain relief was reported from cannabis than from their other medications (Degenhardt et al, 2015). A single case study also reported reduced requirements for breakthrough pain with oral delta-9-THC administration (Holdcroft et al, 1997). Taken together, these reports support the need for high-quality studies to directly assess the opioid-sparing effect of cannabinoids under controlled conditions.

This review identified some limitations in the literature. The pre-clinical studies examined used a range of animal populations, antinociceptive assays, opioids, and cannabinoids, and often had small numbers of animals per group. This resulted in statistical heterogeneity. Despite this, a large and significant effect was observed in the meta-analysis. No studies examined the opioid-sparing effect of cannabidiol alone, in combination with delta-9-THC outside of a $1: 1$ ratio, or with other cannabinoids. Further, the lack of highquality studies in humans investigating the opioid-sparing effect means that the evidence for this is largely limited to pre-clinical studies. A funnel plot was produced and did not provide evidence of publication or small study bias; however, due to the small number of studies in the metaanalysis $(<10)$ the interpretation of the funnel plot is limited. 
The potential for cannabinoids to reduce opioid dose requirements and extend the duration of effective analgesia should not be understated. The rapid increase in opioid use and opioid-associated mortality is largely attributed use of opioids in chronic pain treatment (Chou et al, 2015; Zedler et al, 2014). Use of lower opioid doses has been recommended (Dowell et al, 2016); however, clinical processes to achieve this reduction are not well defined. Opioid-sparing medications may have enormous clinical relevance by enabling effective pain treatment with lower opioid doses and a potential reduction in opioid-related mortality.

In conclusion, pre-clinical studies support the opioidsparing effect of delta-9-THC. However, the findings from clinical trials are inconsistent, with some studies found to have important limitations such as a lack of placebo control. An opioid-sparing effect of cannabinoids in chronic pain patients was observed in only one very-low-quality clinical study. These findings provide an early signal that warrants exploration. It remains to be seen if these promising preclinical and observational findings can be replicated in large, well-designed clinical studies.

\section{FUNDING AND DISCLOSURE}

SN is supported by a NHMRC Research Fellowship (\#1013803). The National Drug and Alcohol Research Centre at the University of New South Wales is supported by funding from the Australian Government under the Substance Misuse Prevention and Service Improvements Grant Fund. The contents of the published material are solely the responsibility of the authors and do not reflect the funding bodies. MAW has received a grant to his institution from CanniMed. BLF has received speaker fees or consulting fees from Allergan, Mettrum, CCIC, Mylan Pharmaceutical, Pfizer, Ethypharm, Richter Pharmaceuticals, and Lundbeck. $\mathrm{He}$ also received salary/grant support from Pfizer and Bioprojet, and in kind support from GW Pharma, Mylan Pharmaceuticals, and Brainsway. SN and NL have been investigators on untied educational grants from ReckittBenckiser. SN and MF have been investigators on an untied education grant from Indivior. KEK has previously received a speaker's honorarium from Pfizer and Mundipharma, in addition to fees from an advisory board and an educational grant from Seqirus. As Director of NDARC, MF notes that the National Drug and Alcohol Research Centre has received untied educational grants from Mundipharma and Indivior. MF took part in a single research advisory board with Indivior in 2014. The remaining authors declare no conflict of interest.

\section{ACKNOWLEDGMENTS}

SN, PS, JMT, MAW, BM, NL, KEK, MF, AS, and BLF were involved in the conceptualization of the work. SN and PS screened the abstracts. SN, PS, and JMT extracted the data and checked the extracted data. BBS transformed the data, prepared the data for meta-analysis, and provided advice on the meta-analysis. SN conducted the meta-analysis and drafted the manuscript with assistance from BM, PS, and JMT. All authors revised the manuscript and approved the final version.

\section{REFERENCES}

Abrams DI, Couey P, Shade SB, Kelly ME, Benowitz NL (2011). Cannabinoid-opioid interaction in chronic pain. Clin Pharmacol Ther 90: 844-851.

Berge O-G (2011). Predictive validity of behavioural animal models for chronic pain. Br J Pharmacol 164: 1195-1206.

Boehnke KF, Litinas E, Clauw DJ (2016). Medical cannabis use is associated with decreased opiate medication use in a retrospective cross-sectional survey of patients with chronic pain. J Pain 17: 739-744.

Cencioni MT, Chiurchiu V, Catanzaro G, Borsellino G, Bernardi G, Battistini L et al (2010). Anandamide suppresses proliferation and cytokine release from primary human T-lymphocytes mainly via CB2 receptors. PLoS ONE 5: e8688.

Chou R, Turner JA, Devine EB, Hansen RN, Sullivan SD, Blazina I et al (2015). The effectiveness and risks of long-term opioid therapy for chronic pain: a systematic review for a National Institutes of Health Pathways to Prevention Workshop Effectiveness and Risks of Long-Term Opioid Therapy for Chronic Pain. Ann Intern Med 162: 276-286.

Cichewicz DL (2004). Synergistic interactions between cannabinoid and opioid analgesics. Life Sci 74: 1317-1324.

Cichewicz DL, Martin ZL, Smith FL, Welch SP (1999). Enhancement mu opioid antinociception by oral delta9-tetrahydrocannabinol: dose-response analysis and receptor identification. J Pharmacol Exp Ther 289: 859-867.

Cichewicz DL, McCarthy EA (2003). Antinociceptive synergy between delta(9)-tetrahydrocannabinol and opioids after oral administration. J Pharmacol Exp Ther 304: 1010-1015.

Cichewicz DL, Welch SP (2003). Modulation of oral morphine antinociceptive tolerance and naloxone-precipitated withdrawal signs by oral Delta 9-tetrahydrocannabinol. J Pharmacol Exp Ther 305: 812-817.

Cichewicz DL, Welch SP, Smith FL (2005). Enhancement of transdermal fentanyl and buprenorphine antinociception by transdermal delta9-tetrahydrocannabinol. Eur J Pharmacol 525: 74-82.

Cox ML, Haller VL, Welch SP (2007). Synergy between delta9tetrahydrocannabinol and morphine in the arthritic rat. Eur $J$ Pharmacol 567: 125-130.

da Fonseca Pacheco D, Klein A, de Castro Perez A, da Fonseca Pacheco CM, de Francischi JN, Duarte ID (2008). The mu-opioid receptor agonist morphine, but not agonists at delta- or kappaopioid receptors, induces peripheral antinociception mediated by cannabinoid receptors. Br J Pharmacol 154: 1143-1149.

Degenhardt L, Lintzeris N, Campbell G, Bruno R, Cohen M, Farrell $\mathrm{M}$ et al (2015). Experience of adjunctive cannabis use for chronic non-cancer pain: findings from the Pain and Opioids IN Treatment (POINT) study. Drug Alcohol Depend 147: 144-150.

Dowell D, Haegerich TM, Chou R (2016). CDC Guideline for Prescribing Opioids for Chronic Pain - United States, 2016. MMWR Recomm Rep 65: 1-49.

Finn DP, Beckett SR, Roe CH, Madjd A, Fone KC, Kendall DA et al (2004). Effects of coadministration of cannabinoids and morphine on nociceptive behaviour, brain monoamines and HPA axis activity in a rat model of persistent pain. Eur J Neurosci 19: 678-686.

Gui H, Tong Q, Qu W, Mao CM, Dai SM (2015). The endocannabinoid system and its therapeutic implications in rheumatoid arthritis. Int Immunopharmacol 26: 86-91.

Guyatt GH, Oxman AD, Vist GE, Kunz R, Falck-Ytter Y, Alonso-Coello P et al (2008). GRADE: an emerging consensus on rating quality of evidence and strength of recommendations. Br Med J 336: 924-926.

Haroutounian S, Ratz Y, Ginosar Y, Furmanov K, Saifi F, Meidan R et al (2016). The effect of medicinal cannabis on pain and quality of life outcomes in chronic pain: a Prospective Open-label Study. Clin J Pain 32: 1036-1043. 
Herkenham M, Lynn AB, Johnson MR, Melvin LS, de Costa BR, Rice KC (1991). Characterization and localization of cannabinoid receptors in rat brain: a quantitative in vitro autoradiographic study. J Neurosci 11: 563-583.

Hohmann AG, Briley EM, Herkenham M (1999). Pre- and postsynaptic distribution of cannabinoid and mu opioid receptors in rat spinal cord. Brain Res 822: 17-25.

Holdcroft A, Smith M, Jacklin A, Hodgson H, Smith B, Newton M et al (1997). Pain relief with oral cannabinoids in familial Mediterranean fever. Anaesthesia 52: 483-486.

Houser SJ, Eads M, Embrey JP, Welch SP (2000). Dynorphin B and spinal analgesia: induction of antinociception by the cannabinoids CP55,940, Delta(9)-THC and anandamide. Brain Res 857: 337-342.

Howlett AC, Barth F, Bonner TI, Cabral G, Casellas P, Devane WA et al (2002). International Union of Pharmacology. XXVII. Classification of cannabinoid receptors. Pharmacol Rev 54: 161-202.

Ibrahim M, Rude M, Stagg N, Mata H, Lai J, Vanderah T et al (2006). CB2 cannabinoid receptor mediation of antinociception. Pain 122: 36-42.

Ibrahim MM, Porreca F, Lai J, Albrecht PJ, Rice FL, Khodorova A et al (2005). CB2 cannabinoid receptor activation produces antinociception by stimulating peripheral release of endogenous opioids. Proc Natl Acad Sci USA 102: 3093-3098.

Jensen B, Chen J, Furnish T, Wallace M (2015). Medical marijuana and chronic pain: a review of basic science and clinical evidence. Curr Pain Headache Rep 19: 50.

Johnson JR, Burnell-Nugent M, Lossignol D, Ganae-Motan ED, Potts R, Fallon MT (2010). Multicenter, double-blind, randomized, placebo-controlled, parallel-group study of the efficacy, safety, and tolerability of THC:CBD extract and THC extract in patients with intractable cancer-related pain. J Pain Symptom Manage 39: 167-179.

Katsuyama S, Mizoguchi H, Kuwahata H, Komatsu T, Nagaoka K, Nakamura $\mathrm{H}$ et al (2013). Involvement of peripheral cannabinoid and opioid receptors in beta-caryophyllene-induced antinociception. Eur J Pain 17: 664-675.

Li JX, McMahon LR, Gerak LR, Becker GL, France CP (2008). Interactions between Delta(9)-tetrahydrocannabinol and mu opioid receptor agonists in rhesus monkeys: discrimination and antinociception. Psychopharmacology 199: 199-208.

Lissoni P, Porro G, Messina G, Porta E, Rovelli F, Roselli MG et al (2014). Morphine, melatonin, Marijuana, Magnolia and MYRRH as the "five $\mathrm{m}$ " schedule in the treatment of cancer pain and the possible dose-dependency of the antitumor and analgesic effects of the pineal hormone melatonin. Anticancer Res 34: 6033-6034.

Litchfield JA, Wilcoxon F (1949). A simplified method of evaluating dose-effect experiments. J Pharmacol Exp Ther 96: 99-113.

Lynch ME, Clark AJ (2003). Cannabis reduces opioid dose in the treatment of chronic non-cancer pain. J Pain Symptom Manage 25: 496-498.

Maguire DR, Yang W, France CP (2013). Interactions between muopioid receptor agonists and cannabinoid receptor agonists in rhesus monkeys: antinociception, drug discrimination, and drug self-administration.(Erratum appears in J Pharmacol Exp Ther. 2014 Mar; 348(3): 490-1 Note: Dosage error in article text). J Pharmacol Exp Ther 345: 354-362.

Manzanares J, Corchero J, Romero J, Fernandez-Ruiz JJ, Ramos JA, Fuentes JA (1999). Pharmacological and biochemical interactions between opioids and cannabinoids. Trends Pharmacol Sci 20: 287-294.

Mason DJ Jr, Lowe J, Welch SP (1999). Cannabinoid modulation of dynorphin A: correlation to cannabinoid-induced antinociception. Eur J Pharmacol 378: 237-248.

Moher D, Liberati A, Tetzlaff J, Altman DG (2009). Preferred reporting items for systematic reviews and meta-analyses: the PRISMA statement. PLoS Med 6: e1000097.
Naef M, Curatolo M, Petersen-Felix S, Arendt-Nielsen L, Zbinden A, Brenneisen R (2003). The analgesic effect of oral delta-9-tetrahydrocannabinol (THC), morphine, and a THC-morphine combination in healthy subjects under experimental pain conditions. Pain 105: 79-88.

Narang S, Gibson D, Wasan AD, Ross EL, Michna E, Nedeljkovic SS et al (2008). Efficacy of dronabinol as an adjuvant treatment for chronic pain patients on opioid therapy. J Pain 9: 254-264.

Pertwee RG (2006). The pharmacology of cannabinoid receptors and their ligands: an overview. Int J Obes 30: S13-S18.

Pertwee RG (2008). The diverse CB1 and CB2 receptor pharmacology of three plant cannabinoids: delta9-tetrahydrocannabinol, cannabidiol and delta9-tetrahydrocannabivarin. Br J Pharmacol 153: 199-215.

Portenoy RK, Ganae-Motan ED, Allende S, Yanagihara R, Shaiova L, Weinstein S et al (2012). Nabiximols for opioidtreated cancer patients with poorly-controlled chronic pain: a randomized, placebo-controlled, graded-dose trial. J Pain 13: 438-449.

Pugh G Jr, Mason DJ Jr, Combs V, Welch SP (1997). Involvement of dynorphin $\mathrm{B}$ in the antinociceptive effects of the cannabinoid CP55,940 in the spinal cord. J Pharmacol Exp Ther 281: 730-737.

Pugh G Jr, Smith PB, Dombrowski DS, Welch SP (1996). The role of endogenous opioids in enhancing the antinociception produced by the combination of DELTA9-tetrahydrocannabinol and morphine in the spinal cord. J Pharmacol Exp Ther 279: 608-616.

Quartilho A, Mata HP, Ibrahim MM, Vanderah TW, Porreca F, Makriyannis A et al (2003). Inhibition of inflammatory hyperalgesia by activation of peripheral CB2 cannabinoid receptors. Anesthesiology 99: 955-960.

Reche I, Fuentes JA, Ruiz-Gayo M (1996). Potentiation of delta 9-tetrahydrocannabinol-induced analgesia by morphine in mice: involvement of mu- and kappa-opioid receptors. Eur J Pharmacol 318: 11-16.

Rice AS, Smith BH, Blyth FM (2015). Pain and the global burden of disease. Pain 157: 791-796.

Roberts JD, Gennings C, Shih M (2006). Synergistic affective analgesic interaction between delta-9- tetrahydrocannabinol and morphine. Eur J Pharmacol 530: 54-58.

Salio C, Fischer J, Franzoni MF, Mackie K, Kaneko T, Conrath M (2001). CB1-cannabinoid and mu-opioid receptor co-localization on postsynaptic target in the rat dorsal horn. NeuroReport 12: 3689-3692.

Seeling W, Kneer L, Buchele B, Gschwend J, Maier L, Nett C et al (2006). DELTA9-tetrahydrocannabinol and the opioid receptor agonist piritramide do not act synergistically in postoperative pain. Anaesthesist 55: 391-400.

Smith FL, Cichewicz D, Martin ZL, Welch SP (1998). The enhancement of morphine antinociception in mice by delta9-tetrahydrocannabinol. Pharmacol Biochem Behav 60: 559-566.

Smith PA, Selley DE, Sim-Selley LJ, Welch SP (2007). Low dose combination of morphine and delta9-tetrahydrocannabinol circumvents antinociceptive tolerance and apparent desensitization of receptors. Eur J Pharmacol 571: 129-137.

Smith PB, Welch SP, Martin BR (1994). Interactions between delta 9-tetrahydrocannabinol and kappa opioids in mice. J Pharmacol Exp Ther 268: 1381-1387.

Tham SM, Angus JA, Tudor EM, Wright CE (2005). Synergistic and additive interactions of the cannabinoid agonist CP55,940 with $\mathrm{mu}$ opioid receptor and alpha2-adrenoceptor agonists in acute pain models in mice. Br J Pharmacol 144: 875-884.

Valverde O, Noble F, Beslot F, Dauge V, Fournie-Zaluski MC, Roques BP (2001). Delta9-tetrahydrocannabinol releases and facilitates the effects of endogenous enkephalins: reduction in 
morphine withdrawal syndrome without change in rewarding effect. Eur J Neurosci 13: 1816-1824.

Vigano D, Rubino T, Parolaro D (2005). Molecular and cellular basis of cannabinoid and opioid interactions. Pharmacol Biochem Behav 81: 360-368.

Volkow ND, McLellan AT (2016). Opioid abuse in chronic pain misconceptions and mitigation strategies. $N$ Engl J Med 374: 1253-1263.

Wakley AA, Craft RM (2011). THC-methadone and THCnaltrexone interactions on discrimination, antinociception, and locomotion in rats. Behav Pharmacol 22: 489-497.

Walker JM, Hohmann AG, Martin WJ, Strangman NM, Huang SM, Tsou K (1999). The neurobiology of cannabinoid analgesia. Life Sci 65: 665-673.

Walker JM, Huang SM (2002). Cannabinoid analgesia. Pharmacol Ther 95: 127-135.

Ware MA, Fitzcharles M-A, Joseph L, Shir Y (2010a). The effects of nabilone on sleep in fibromyalgia: results of a randomized controlled trial. Anesth Analg 110: 604-610.

Ware MA, Wang T, Shapiro S, Robinson A, Ducruet T, Huynh T et al (2010b). Smoked cannabis for chronic neuropathic pain: a randomized controlled trial. CMAJ 182: E694-E701.

Welch SP, Stevens DL (1992). Antinociceptive activity of intrathecally administered cannabinoids alone, and in combination with morphine, in mice. J Pharmacol Exp Ther 262: $10-18$.

Williams IJ, Edwards S, Rubo A, Haller VL, Stevens DL, Welch SP (2006). Time course of the enhancement and restoration of the analgesic efficacy of codeine and morphine by 89-tetrahydrocannabinol. Eur J Pharmacol 539: 57-63.

Williams J, Haller VL, Stevens DL, Welch SP (2008). Decreased basal endogenous opioid levels in diabetic rodents: effects on morphine and delta-9-tetrahydrocannabinoid-induced antinociception. Eur J Pharmacol 584: 78-86.

Wilson AR, Maher L, Morgan MM (2008). Repeated cannabinoid injections into the rat periaqueductal gray enhance subsequent morphine antinociception. Neuropharmacology 55: 1219-1225.

Woodhams SG, Sagar DR, Burston JJ, Chapman V (2015). The role of the endocannabinoid system in pain. Handb Exp Pharmacol 227: 119-143.

Yesilyurt O, Dogrul A, Gul H, Seyrek M, Kusmez O, Ozkan Y et al (2003). Topical cannabinoid enhances topical morphine antinociception. Pain 105: 303-308.

Zedler B, Xie L, Wang L, Joyce A, Vick C, Kariburyo F et al (2014). Risk factors for serious prescription opioid-related toxicity or overdose among veterans health administration patients. Pain Med 15: 1911-1929.

Supplementary Information accompanies the paper on the Neuropsychopharmacology website (http://www.nature.com/npp) 